\title{
Joint Throughput Optimization for Wireless Mesh Networks
}

\author{
Xiang-Yang Li*, Senior Member, IEEE, Ashraf Nusairat*, Yanwei $\mathrm{Wu}^{*}$, Student Member, IEEE, Yong $\mathrm{Qi}^{\dagger}$, \\ JiZhong Zhao ${ }^{\dagger}$, Xiaowen $\mathrm{Chu}^{\ddagger}$ Member, IEEE, and YunHao Liu Senior Member, IEEE,
}

\begin{abstract}
In this paper, we address the problem of joint channel assignment, link scheduling and routing for throughput optimization in wireless networks with multi-radio and multichannels. We mathematically formulate this problem by taking into account the interference, the number of available radios the set of usable channels and other resource constraints at nodes. We also consider the possible combining of several consecutive channels into one so that a network interface card (NIC) can use the channel with larger range of frequencies and thus improve the channel capacity. Furthermore, we consider several interference models and assume a general yet practical network model in which two nodes may still not communicate directly even if one is within the transmission range of the other. We designed efficient algorithm for throughput (or fairness) optimization by finding flow routing, scheduling of transmissions, and dynamic channel assignment and combining. We show that the performance, fairness and throughput, achieved by our method is within a constant factor of the optimum. Our model also can deal with the situation when each node will charge a certain amount for relaying data to a neighboring node and each flow has a budget constraint. Our extensive evaluation shows that our algorithm can effectively exploit the number of channels and radios. In addition, it shows that combining multiple channels and assigning them to a single user at some time slots indeed increases the maximum throughput of the system compared to assigning a single channel.
\end{abstract}

Index Terms-Wireless mesh networks, multi-channels, multiradios, cross-layer, scheduling, channel assignment, routing, channel combining.

\section{INTRODUCTION}

Wireless mesh networks are being used as the last mile for extending the Internet connectivity for mobile nodes. Many US cities (e.g., Medford, Oregon; Chaska, Minnesota; Nashville, Illinois; and Gilbert, Arizona) have already deployed mesh networks. For wireless mesh networks or sensor networks, the aggregate traffic load of each routing node changes infrequently. The antenna is omni-directional, so the signal sent

${ }^{*} \mathrm{Li}$, Nusairat, and $\mathrm{Wu}$ are with Department of Computer Science, Illinois Institute of Tech., USA. Email: xli@cs.iit.edu, nusaasheit.edu, ywu24eit.edu. '†i and Zhao are with Department of Computer Science and Tech., Xi'an Jiaotong Univ., China, Email: $\{q i y, z j z\} @ m a i l . x j t u . e d u . c n .{ }^{\ddagger}$ Chu is with Department of Computer Science, Hong Kong Baptist Univ., Hong Kong, Email:chxwecomp.hkbu.edu.hk. Liu is with Department of Computer Science and Engineering, Hong Kong Univ. of Science and Tech., Hong Kong, Email:liu@cse.ust.hk. Li is an adjunct professor of Dept. of Computer Science and Tech., Xi'an Jiaotong Univ. The research of Xiang-Yang Li is partially supported by NSF CNS-0832120, and Hong Kong CERG under Grant PolyU-5232/07E. Liu and X.-Li are partially supported by National Basic Research Program of China (973 Program) under grant No. 2006CB30300, the National High Tech. Research and Development Program of China (863 Program) under grant No. 2007AA01Z180, and Hong Kong RGC HKUST 6169/07. Yong Qi and X.-Li are partially supported by National Natural Science Foundation of China under Grant No. 60828003. Xiaowen Chu and X.-Li are partially supported by the RGC under Grant HKBU 2104/06E. by a wireless terminal will be received by all other terminals within its transmission range, and may cause interference to some terminals which are not the intended receivers. In other words, the communication channels are shared by the wireless terminals. Thus, one of the major problems facing wireless networks is the reduction of capacity due to interference caused by simultaneous transmissions. Using multiple channels and multiple radios can alleviate interference because the probability of neighboring transmitting nodes using the same channels decreases, due to the availability of more radios and more channels to be scheduled at a certain time slot.

Recently, several papers [6], [17] studied the capacity of wireless networks under various interference models. Kyasanur and Vaidya [15], [16] studied the capacity region on random multi-hop multi-radio multi-channel wireless networks when there are total $c$ channels available and each node has $m \leq c$ wireless interfaces (radios). Several papers [2], [10] recently studied how to satisfy a certain traffic demand vector from all wireless nodes by a joint routing, link scheduling, and channel assignment under certain wireless interference models.

In this paper, we study the throughput optimization (under certain fairness constraints) via joint routing, link scheduling, and dynamic channel assignment for a given multi-channel multi-radio multi-hop wireless network with multiple sink nodes. Assume that we want to maximize the uploading flow rate (or downloading flow rate) for a source node, or a set of source nodes. To achieve this, we need decide which subset of sink nodes to use for each source node; which paths to route the flow; whether a node should be active at a time-slot and if so then with which neighbor to communicate, using which channel(s) and which interface card(s) at every time-slot. Unlike those previous methods, which often assumed all channels can be used by every wireless interface card, we assume that each wireless node can operate on a subset of known channels because of either the availability of the channels in the neighborhood when spectrum is used opportunistically or the constraints due to the wireless interface cards. Each wireless interface is capable of dynamically switching channels between different time slots. Further, we study this problem under a variety of interference models and assumed that different nodes may have different transmission radii and interference radii in contrast to [2] where uniform interference range is assumed to be a fixed multiple of the uniform communication range. We prove a constant approximation ratio of our method for the variety of interference models we studied. We also studied the case when some wireless cards are capable of combining multiple consecutive channels into a single channel between different time slots [9]. We studied this problem under two different 
channel combining conditions: (1) with channel-combining and (2) without channel-combining, and studied the effect of channel-combining on the maximum throughput (under certain fairness constraints). We also studied the scenario when each intermediate node $u$ will charge a fixed cost, say $\mathbf{p}(u, v)$, for each packet relayed to a neighboring node $v$; and each flow from a source $s$ to a target node $t$ has a budget $\mathbf{B}(s, t)$. Our algorithms will approximately maximize the total flow while the total cost is within the budget constraint. In summary, the main contributions of this paper are follows.

(1) Theoretical Performance Guarantee for Algorithms: We consider four kinds of interference models: RTS/CTS model and protocol interference model with fixed transmission power (fPrIM), protocol interference model (PrIM), transmitter model (TxIM)). For all models, we use a novel flow technique to simultaneously decide a multi-path routing for each traffic demand, choose the gateway nodes where the traffic will use, and design efficient link scheduling algorithms that can achieve fairness or throughput at most a constant factor of the optimum. To the best of our knowledge, we are the first one to prove asymptotic optimal bounds for the performance. To study the maximal achievable fairness or throughput by a given fixed wireless network, we apply a cross-layer approach integrating the routing, TDMA node/link scheduling, and dynamic channel switching/combining. We formulate the problem of routing and link-channel assignment as a mixed integer problem and relax it to a linear programming. We then present a sufficient condition for flows that can be scheduled by a certain efficient link-channel scheduling algorithm. Combined with other similar necessary conditions for schedulable flows, we prove that the fairness or the throughput in our scheduling is within a constant factor of the optimum.

(2) Impact of Channel-Combining: We combine multiple consecutive channels to study its impact on fairness and maximum throughput of the wireless mesh network. We assume that, the radio is capable of combining multiple consecutive channels into one combined-channel in a negligible time compared to the time slot duration. The combined-channel bandwidth is close to the sum of all channels' bandwidth that are combined. Softransceiver, a software defined radio (SDR) developed by BitWave Semiconductor Inc., described in [9] achieves channel-combining by changing the channel bandwidth in real-time. It can shift the center frequency, modify the bandwidth and sampling rate, and change the linearity and noise figure of a transceiver channel in real time. To the best of our knowledge, we are the first to study channel-combining in wireless mesh networks. Our extensive evaluation shows that channel-combining increases the fairness and the maximal throughput of the network compared to that without channel combining.

(3) Realistic Models and Other Restrictions: We address the routing, link-channel scheduling in a more realistic networking model. The only requirements for our theoretical performance guarantee are that (1) if $\|v-u\| \leq R_{I}(u)$ for a sender $u$, then the transmission by $u$ will always interfere the reception of node $v$ that is not intended receiver; (2) if receiver $v$ can receive data from $u$ directly then $\|v-u\| \leq R_{T}(u)$, where $R_{T}(u)<R_{I}(u)$. Further we considered throughput optimization under other possible constraints such as 1) each node may have a certain resource constraints (e.g., energy) and each relaying will spend a certain amount of the resource; 2) each node may charge a certain amount for relay data to a neighbor node and each source node has a budget constraint. We provide theoretical bounds for link-scheduling algorithms in all interference models and constraints we consider. Our extensive evaluation shows that our algorithm can effectively exploit the available channels and radios (NICs) at each node, and it performs much better than the theoretical bounds.

The rest of the paper is organized as follows. In Section II, we present our network model and the problem to be studied. We mathematically formulate the problem in Section III and provide an efficient link and channel scheduling method in Section IV. Then we report our simulation results in Section $\mathrm{V}$ and review the related works in Section VI. We conclude our paper in Section VII.

\section{System Model And Assumption}

\section{A. Network System Models}

Assume that there is a set $V$ of $n$ communication terminals deployed in a plane. Its complete communication graph is defined as a directed graph $G=(V, E)$, where $V=\left\{v_{1}, \ldots, v_{n}\right\}$ is the set of terminals and $E$ is the set of possible directed communication links. Let $\mathbf{E}^{-}(u)$ denote the set of directed links that end at node u, i.e. $(w, u)$; and $\mathbf{E}^{+}(u)$ denote the set of directed links that start at node $u$, i.e. $(u, v)$. Every terminal $v_{i}$ has a transmission range $R_{T}(i)$ such that the necessary condition for a terminal $v_{j}$ to receive correctly the signal from $v_{i}$ is $\left\|v_{i}-v_{j}\right\| \leq R_{T}(i)$, where $\left\|v_{i}-v_{j}\right\|$ is the Euclidean distance between $v_{i}$ and $v_{j}$. Notice that $\left\|v_{i}-v_{j}\right\| \leq R_{T}(i)$ is not the sufficient condition for $\left(v_{i}, v_{j}\right) \in E$. Some links do not belong to $G$ because of either the physical barriers or the selection of routing protocols. To the best of our knowledge, only [13], [27] used the similar model as ours. We always use $\mathbf{L}_{i, j}$ to denote the directed link $\left(v_{i}, v_{j}\right)$ hereafter. Each terminal $v_{i}$ also has an interference range $R_{I}(i)$ such that terminal $v_{j}$ is interfered by the signal from $v_{i}$ whenever $\left\|v_{i}-v_{j}\right\| \leq R_{I}(i)$ and $v_{j}$ is not the intended receiver. Typically, $c_{1} R_{T}(i)<R_{I}(i) \leq c_{2} \cdot R_{T}(i)$ for some constants $1<c_{1}, c_{2}$. In practice, $2 \leq c_{1}, c_{2} \leq 4$. For all wireless nodes, let $\gamma=\max _{v_{i} \in V} \frac{R_{I}(i)}{R_{T}(i)}$.

We assume that the wireless mesh network (WMN) is multi-hop, multi-radio, and multi-channel network (MMM). Let $\mathbb{F}=\left\{\mathbf{f}_{1}, \mathbf{f}_{2}, \cdots, \mathbf{f}_{K}\right\}$ be the set of $K$ orthogonal channels (typically frequency channels or CDMA codes) that can be used by all wireless nodes. For example, for 802.11 networks, $K=11$. Each wireless terminal $u$ is equipped with $\mathcal{I}(u) \geq 1$ radio interfaces, namely $\kappa(u, 1), \kappa(u, 2), \cdots, \kappa(u, \mathcal{I}(u))$. We assume that each radio interface $\kappa(u, i)$ is capable of combining set of consecutive channels $\mathbf{f}_{a}, \mathbf{f}_{a+1}, \cdots, \mathbf{f}_{b}$ into one combined-channel $\mathbf{f}_{a b}$, where $a \leq b \leq K$. It is worth noting that the combined-channel $\mathbf{f}_{a b}$ bandwidth is not necessarily equal to the sum of the combined channels $\mathbf{f}_{a}$ and $\mathbf{f}_{b}$ due to frequency overlapping of the channels, for example in 802.11 (USA, 2.4GHz) there are 11 channels: channels 1,6 , and 11 are non-overlapping, combining channels 1 and 6 leads to 
double the bandwidth, while channels 1 and 4 are overlapping and combining them does not lead to double the bandwidth it only increases the combined-channel bandwidth by $15 \mathrm{MHz}$. Channel combining allows the scheduler to assign multiple channels on a radio interface at a given time slot $t$ to a single NIC. The scheduler will dynamically change the number of combined channels assigned to a user to achieve fairness and at the same time provide the user with higher throughput. In the case $a=b$ the scheduler will assign one channel to the user and no channel combining takes place at the radio interface. Let $\mathcal{F}=\left\{\mathbf{f}_{a_{1} b_{1}}, \mathbf{f}_{a_{2} b_{2}}, \cdots, \mathbf{f}_{a_{m} b_{m}}\right\}$ be the set of $m$ (not necessarily pairwise orthogonal) channels that can be used by all wireless nodes, where $b_{i} \geq a_{i} \forall i \in[1, m]$ and $m \leq K(K+1) / 2 . \mathcal{F}$ includes both combined and noncombined channels.

For the combined-channels we denote the co-combinedchannel interference by $\mathbf{I}_{s e t}\left(\mathbf{f}_{a b}, \mathbf{f}_{p q}\right)$ and is defined as:

$$
\mathbf{I}_{s e t}\left(\mathbf{f}_{a b}, \mathbf{f}_{p q}\right)= \begin{cases}1 & \text { if } \mathbf{f}_{a b} \text { and } \mathbf{f}_{p q} \text { interfere } \\ 0 & \text { otherwise }\end{cases}
$$

For example, when no adjacent channel interference is defined, we have $\mathbf{f}_{a b}$ and $\mathbf{f}_{p q}$ do not interfere iff they are disjoint. Assume that $\mathbf{I}_{s e t}\left(\mathbf{f}_{a b}, \mathbf{f}_{p q}\right)$ is given for all pairs of $\mathbf{f}_{a b}$ and $\mathbf{f}_{p q}$.

In the literature (e.g., [2], [10], [13]) it is often assumed that a wireless interface card can operate on all channels $\mathbb{F}$, which is not true always. We assume a general case that each wireless interface can only operate on a subset of channels from $\mathbb{F}$ due to the hardware constraints and the same assumption is valid for $\mathcal{F}$. More specifically, we let $\mathcal{F}(u, i)$ be the set of channels (combined and non-combined) that can be used by the $i$ th wireless interface $\kappa(u, i)$ for node $u$, where $1 \leq i \leq \mathcal{I}(u)$.

Let $\delta\left(u, i, \mathbf{f}_{a b}\right) \in\{0,1\}$ be the indicator function whether the $i$ th wireless interface of node $u$ can use channel $\mathbf{f}_{a b}$ or not. Thus, the channels that can be used by a wireless node $u$ is represented by a subset $\mathcal{F}(u) \subset \mathcal{F}$, where $\mathcal{F}(u)=$ $\bigcup_{1 \leq i \leq \mathcal{I}(u)} \mathcal{F}(u, i)$. We also define $\delta\left(u, \mathbf{f}_{a b}\right) \in\{0,1\}$ as the indicator function whether node $u$ can use channel $\mathbf{f}_{a b}$ or not. Obviously, $\delta\left(u, \mathbf{f}_{a b}\right)=\bigvee \delta\left(u, i, \mathbf{f}_{a b}\right), \forall i \in[1, \mathcal{I}(u)]$.

For notational convenience, we use $\mathcal{F}(e)$ to denote the set of common channels among $\mathcal{F}(u)$ and $\mathcal{F}(v)$ for any link $e=$ $(u, v)$, and $\delta\left(e, \mathbf{f}_{a b}\right) \in\{0,1\}$ be the indicator function whether a channel $\mathbf{f}_{a b}$ can be used by a link $e$. Obviously, $\delta\left(e, \mathbf{f}_{a b}\right)=$ $\delta\left(u, \mathbf{f}_{a b}\right) \cdot \delta\left(v, \mathbf{f}_{a b}\right)$ for a link $e=(u, v)$.

For each link $e=(u, v)$ operating on a channel $\mathbf{f}_{a b} \in \mathcal{F}(e)$, we denote by $\mathbf{c}\left(e, \mathbf{f}_{a b}\right)$ the rate for link $e$. This is the maximum rate at which mesh node $u$ can communicate with mesh node $v$ in one-hop communication using channel $\mathbf{f}_{a b}$. Note, we do not assume any relations between the data rate $\mathbf{c}\left(e, \mathbf{f}_{a b}\right)$ of a combined-channel, and the data rates of all individual channels in the combined-channel. Clearly the maximum rate that can be supported by a link $e$ is at most $\sum_{\mathbf{f}_{a b} \in \mathcal{F}(e)} \mathbf{c}\left(e, \mathbf{f}_{a b}\right)$. Notice that the links are directed, thus, the capacity could be asymmetric, i.e., $\mathbf{c}\left((u, v), \mathbf{f}_{a b}\right)$ may not same as $\mathbf{c}\left((v, u), \mathbf{f}_{a b}\right)$.

Our approach is to visualize a multi-radio node as a collection of fully-connected multiple virtual nodes with infinite bandwidth links between them, i.e., node $u$ with $\mathcal{I}(u)$ radio interfaces can be seen as a group of $\mathcal{I}(u)$ fully-connected virtual nodes $\widehat{u_{i}}, i \in[1, \mathcal{I}(u)]$. Each virtual node $\widehat{u_{i}}$ has exactly radio interface $i$ to connect to neighboring virtual nodes $\widehat{v_{k}}$. See Figure 1 for illustration of an example in which $\mathcal{I}(u)=2$, $\mathcal{I}(w)=1, \mathcal{I}(v)=2$ and $\mathcal{I}(z)=3$. We define two types of virtual links for each virtual node $\widehat{u_{i}}$ :

1) directed external virtual links that connect virtual node $\widehat{u_{i}}$ with other nodes outside its group, e.g., virtual node $\widehat{v_{k}}$. This type of link has limited capacity and may cause interference to other external virtual links using the same channel.

2) internal virtual links that connect virtual node $\widehat{u_{i}}$ with all virtual nodes $\widehat{u_{q}}$ in its group, where $q \neq i$ and $q \in$ $[1, \mathcal{I}(u)]$. This link has infinite capacity, does not interfere with any other link, and resembles the internal switching of data from one radio interface to another radio interface by a node.

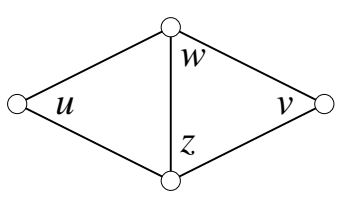

(a) original network

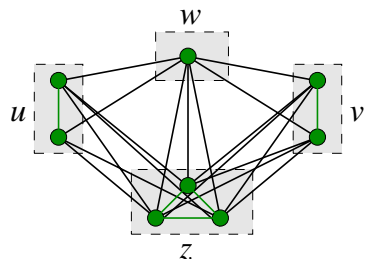

(b) virtual nodes and links
Fig. 1. Virtual nodes and virtual links defined by splitting nodes. The virtual nodes in one shaded region correspond to a node in the original network.

Now, we define directed link $e(u, v)$ as the superposition of directed virtual links $\widehat{e}_{i j}(u, v)$, where $\widehat{e}_{i j}$ is defined as the external virtual link from $\widehat{u_{i}}$ to $\widehat{v_{j}}$ using radio interface $i$ of node $u$ and $j$ of node $v$.

We define $\delta\left(\widehat{e}_{i j}, \mathbf{f}_{a b}\right) \in[0,1]$ as the indicator function whether virtual link $\widehat{e}_{i j}$ can use $\mathbf{f}_{a b}$ for communication. Let $\mathbb{R} c c_{i j}$ be the set of common channels between radio interface $i$ of node $u$ and radio interface $j$ of node $v$.

Then $\widehat{e}_{i j}$ can have up to $\left|\mathbb{R} c c_{i j}\right|$ choices of channels for communication. In a TDMA link scheduling, for each virtual link $\widehat{e}_{i j}=(u, v)$, we denote by $\mathbf{c}\left(\widehat{e}_{i j}, \mathbf{f}_{a b}\right)$ the capacity of the virtual link using channel $\mathbf{f}_{a b}$. For simplicity, we also use $\widehat{e}$ to denote such a communication link. We denote the fraction of time virtual link $\widehat{e}$ will be actively using $\mathbf{f}_{a b}$ by $\alpha\left(\widehat{e}, \mathbf{f}_{a b}\right)$, denote the capacity of $\widehat{e}$ using $\mathbf{f}_{a b}$ as $\mathbf{c}\left(\widehat{e}, \mathbf{f}_{a b}\right)$, denote the subset of channels that can be used by $\widehat{e}$ as $\mathbb{R} c c(\widehat{e})$, denote the set of directed external virtual links that end at node $u$ as $\widehat{\mathbf{E}}^{-}(u)$ and denote the set of directed external virtual links that start at node $u$ as $\widehat{\mathbf{E}}^{+}(u)$.

We also assume that among the set $V$ of all wireless nodes, some of them have gateway functionality and provides the connectivity to the Internet. For simplicity, let $\mathcal{S}=$ $\left\{\mathbf{s}_{1}, \mathbf{s}_{2}, \cdots, \mathbf{s}_{g}\right\}$ be the set of $g$ gateway nodes, where $\mathbf{s}_{i}$ is actually node $v_{n+i-g}$. All other wireless nodes $v_{i}$ (for $1 \leq i \leq n-g)$ are called ordinary wireless nodes.

We assume that the gateway nodes will not act as relay node for a pair of ordinary wireless nodes. Each ordinary node $u$ will aggregate the traffic from all its users and then route them to the Internet through some gateway nodes. We use $\ell_{O}(u)$ to denote the total aggregated outgoing traffic of node $u$ users and $\ell_{I}(u)$ to denote the total aggregated incoming traffic of node $u$ users. We will mainly concentrate on incoming traffic in this 
paper. For notation simplicity, we use $\ell(u)$ to denote such load for node $u$. Notice that the traffic $\ell(u)$ is not requested to be routed through a specific gateway node, neither requested to be using a single routing path. But we require that the traffic generated by a specific user, which is subset of $\ell(u)$, be routed through the same route to avoid flow splitting problems. In this paper we assume the nodes are highly available and reliable, therefor we do not need to address route recovery mechanisms when a node fails. Our results can be easily extended to deal with both incoming and outgoing traffic by defining routing flows for both traffics separately.

There are a number of distinctions of the model used here with the models used in previous study: We do not require the same transmission range (also same interference range) for every wireless node; We do not require the communication graph to be complete, i.e., some communication links may not exist due to barriers or may be not used by routing selection; We assume that, at each time slot, the radio can combine multiple consecutive channels into a single combined-channel; We also consider a more wide list of constraints such as budget constraints by flow, and resource constraints by relay nodes.

\section{B. Interference Models}

To schedule two links at the same time slot, we must ensure that the schedule will avoid the interference. Two different types of interference have been studied in the literature, namely, primary interference and secondary interference. In addition to these interferences, there could be some other constraints on the scheduling, e.g., the radio networks that deploy the IEEE 802.11 protocol with request-to-send and clear-to-send (RTS/CTS) mechanism will pose some additional constraints. Several different interference models have been used to model the interferences in wireless networks. We briefly review them in the following.

Protocol Interferences Model (PrIM): It was first proposed in [7]. In this model, a transmission by a node $v_{i}$ is successfully received by a node $v_{j}$ iff the intended destination $v_{j}$ is sufficiently apart from the source of any other simultaneous transmission, i.e., $\left\|v_{k}-v_{j}\right\| \geq(1+\eta)\left\|v_{i}-v_{j}\right\|$ for any node $v_{k} \neq v_{i}$. Here constant $\eta>0$ models situations where a guard zone is specified by the protocol to prevent a neighboring node from transmitting on the same channel at the same time. This model was used in [13] to study the throughput optimization for wireless networks.

Fixed Protocol Interferences Model (fPrIM): This was used in [27] to study link scheduling for mesh networks. They assumed that a node will not dynamically change its power based on the intended receiver in a packet-level and each node $v_{i}$ has its own fixed transmission power and thus fixed transmission range $R_{T}(i)$ and fixed interference range $R_{I}(i)$. Here any node $v_{j}$ will be interfered by the signal from $v_{k}$ if $\left\|v_{k}-v_{j}\right\| \leq R_{I}(k)$ and node $v_{k}$ is sending signal to some node other than $v_{j}$.

RTS/CTS Model: This model was also studied previously, e.g., [2]. When using RTS/CTS mechanism, a transmitter first sends a RTS frame before sending a data frame. The intended receiver then responds with a CTS frame indicating that the transmitter can send the data frame. Within the CTS frame, the receiver provides a value in the duration field of the frame header for holding off other stations from transmitting for a certain time. For every pair of transmitter and receiver, all nodes that are within the interference range of either the transmitter or the receiver cannot transmit. The interference region, denoted by $I_{i, j}$, of a link $\mathbf{L}_{i, j}$ is the union of the interference region of nodes $v_{i}$ and $v_{j}$. When a directed link $v_{i} v_{j}$ (or $v_{j} v_{i}$ ) is active, all simultaneous transmitting links $v_{p} v_{q}$ cannot have an end-point inside the area $I_{i, j}$.

Transmitter Interference Model (TxIM): This model was introduced by Yi et al. [28]. Here a transmission from a node $v_{i}$ is successful if and only if, for any other transmitter $v_{k}$, $d\left(v_{i}, v_{k}\right) \geq(1+\eta)\left(R_{T}(i)+R_{T}(k)\right)$, where $\eta$ is a system parameter.

It is known that joint routing, link scheduling and channel assignment will improve the overall network throughput performance. Traditionally, in wired networks, the maximum throughput can be found via the simple maximum flow solution for unicast. This technique cannot be directly applied to wireless networks because that the wireless interference may cause some flow un-schedulable. Further complicating the study is that the channel used by a wireless interface of a node could be dynamically or statically assigned.

To study this cross-layer optimization, we model the interference of multi-channel multi-radio multi-hop wireless networks by a conflict graph. Given a communication graph $G=(V, E)$, we use the conflict graph (e.g., [8]) $F_{G}$ to represent the interference in $G$. Each vertex (denoted by $\left.\mathbf{L}_{i, j}\right)$ of $F_{G}$ corresponds to a directed link $\left(v_{i}, v_{j}\right)$ in the communication graph $G$. There is an edge between vertex $\mathbf{L}_{i, j}$ and vertex $\mathbf{L}_{p, q}$ in $F_{G}$ if and only if $\mathbf{L}_{i, j}$ conflicts with $\mathbf{L}_{p, q}$ due to interference. Recall that whether two links conflict at a certain time depends on (1) the channels used by them for communication, (2) the geometry locations of these two links, and (3) the interference model used underneath, e.g., protocol interference model or RTS/CTS model.

\section{Problem Formulation for Cross-Layer OPTIMIZATION}

In this section, we first give a mixed Integer Programming formulation of the necessary and sufficient conditions when we want to maximize the network throughput or the fairness amongst the flows. For cross-layer optimization, the flow that can be supported by mesh networks not only need to satisfy the capacity constraint, but also need to be schedulable by all links without interference. Furthermore, the scheduling of links in multi-channel and multi-radio mesh networks also need satisfy the channel and radio constraints no matter whether dynamic channel assignment or static channel assignment is used.

\section{A. Maximize Fairness}

Assume that we are given a multi-radio multi-channel multi-hop wireless network $G=(V, E)$ and a flow demand $\ell(u)$ from each source node $u$. Our objective is to find a flow assignment that maximizes the fairness of the achieved flow. Given a routing (and corresponding link and channel 
scheduling), the achieved fairness $\lambda$ is defined as the minimum ratio of achieved flow over the demanded load over all wireless mesh routers. We note that there are many other definitions of flow fairness. For simplicity, we focus on min-fairness here.

If virtual link $\widehat{e}$ is assigned channel $\mathbf{f}_{a b}$ for $\alpha\left(\widehat{e}, \mathbf{f}_{a b}\right)$ fraction of time, then $\alpha\left(\widehat{e}, \mathbf{f}_{a b}\right) \cdot \mathbf{c}\left(\widehat{e}, \mathbf{f}_{a b}\right)$ is the corresponding achieved flow. Clearly, the achieved flow at a router $u$ is the difference between the flow going out of node $u$ and the flow coming to node $u$, i.e., $\sum_{\widehat{e} \in \widehat{\mathbf{E}}^{+}{ }_{(u)}} f(\widehat{e})-\sum_{\widehat{e} \in \widehat{\mathbf{E}}^{-}(u)} f(\widehat{e})$. Here variable $f(\widehat{e})$ is the total scheduled traffics over virtual link $\widehat{e}$ using various channels. We also assume that for each sink $\mathbf{s}_{i}$, its maximum outgoing capacity is $F_{I S P}\left(\mathbf{s}_{i}\right)$, which could be infinity. We use variable $f_{i s p}\left(\mathbf{s}_{i}\right)$ to denote the actual flow out of this sink $\mathbf{s}_{i}$. We add a virtual sink node $v_{0}$ to get $V^{\prime}=V \cup\left\{v_{0}\right\}$ and virtual edges $\left(\mathbf{s}_{i}, v_{0}\right.$ to $G$ to get a communication graph $G^{\prime}$ with capacity $F_{I S P}\left(\mathbf{s}_{i}\right)$. These links will not conflict with any other edge. Then it is easy to get the following Mixed IP for maximizing the fairness.

$$
\left\{\begin{array}{rlll}
\text { MAX Fairness LP: } & \max \lambda & \\
\sum_{\widehat{e} \in \widehat{\mathbf{E}}^{+}(u)} f(\widehat{e})-\sum_{\widehat{e} \in \widehat{\mathbf{E}}^{-}(u)} f(\widehat{e}) & \geq & \lambda \ell(u) & \forall u \in V \\
\sum_{\mathbf{f}_{a b} \in \mathbb{R} c c(\widehat{e})} \alpha\left(\widehat{e}, \mathbf{f}_{a b}\right) \cdot \mathbf{c}\left(\widehat{e}, \mathbf{f}_{a b}\right) & = & f(\widehat{e}) & \forall \widehat{e} \\
\alpha\left(\widehat{e}, \mathbf{f}_{a b}\right) & \geq & 0 & \forall e \\
\sum_{\widehat{e} \in e} \alpha\left(\widehat{e}, \mathbf{f}_{a b}\right) & \leq & 1 & \forall e \\
f_{i s p}\left(\mathbf{s}_{i}\right) & \geq & 0 & \forall \mathbf{s}_{i} \\
f_{i s p}\left(\mathbf{s}_{i}\right) & \leq & F_{I S P}\left(\mathbf{s}_{i}\right) & \forall \mathbf{s}_{i}
\end{array}\right.
$$

The first condition indicates that we need to maximize the load offered by node $u$ (traffic of all node $u$ users). Notice that for nodes without traffic demand, $\ell(u)=0$, thus, first condition implies flow conservation. For a sink node $\mathbf{s}_{i} \in V$, $\sum_{\widehat{e} \in \widehat{\mathbf{E}}^{+}\left(\mathbf{s}_{i}\right)} f(\widehat{e})=f_{i s p}\left(\mathbf{s}_{i}\right) \leq F_{I S P}\left(\mathbf{s}_{i}\right)$. The second condition defines the achieved flow of external virtual link $\widehat{e}$. The third and forth conditions set the upper and lower limits of the fraction of time a channel can be assigned to an external virtual link $\widehat{e}$. The last two conditions set the upper and lower bound on the outgoing flow at the gateway nodes.

\section{B. Maximize Throughout}

In majority applications, we do not only have to guarantee certain fairness of the achieved flows for all end wireless devices, but also have to achieve the largest possible throughput under certain fairness constraints. Assume that we have a minimum fairness constraints $\lambda_{0} \geq 0$. The maximum throughput routing is equivalent to solve the following linear programming (LP-Flow-throughput) for $\alpha\left(\widehat{e}, \mathbf{f}_{a b}\right)$ such that

$$
\begin{array}{rlrl}
\text { LP-Flow-throughput: } \max \sum_{i=1}^{g} f_{i s p}\left(\mathbf{s}_{i}\right) & \\
\sum_{\widehat{e} \in \widehat{\mathbf{E}}^{+}(u)} f(\widehat{e})-\sum_{\widehat{e} \in \widehat{\mathbf{E}}^{-}(u)} f(\widehat{e}) & =f(u) & \forall u \in V-\mathcal{S} \\
f(u) & \geq \lambda_{0} \ell(u) & \forall u \in V \\
\sum_{\widehat{e} \in \widehat{\mathbf{E}}^{-}\left(\mathbf{s}_{i}\right)} f(\widehat{e})+f\left(\mathbf{s}_{i}\right) & =f_{i s p}\left(\mathbf{s}_{i}\right) & \forall \mathbf{s}_{i} \in \mathcal{S} \\
\sum_{\mathbf{f}_{a b} \in \mathbb{R} c c(\widehat{e})} \alpha\left(\widehat{e}, \mathbf{f}_{a b}\right) \cdot \mathbf{c}\left(\widehat{e}, \mathbf{f}_{a b}\right) & =f(\widehat{e}) & \forall \widehat{e} \\
\alpha\left(\widehat{e}, \mathbf{f}_{a b}\right) & \geq 0 & \forall e \\
\sum_{\widehat{e} \in e} \alpha\left(\widehat{e}, \mathbf{f}_{a b}\right) & \leq 1 & \forall e \\
f_{i s p}\left(\mathbf{s}_{i}\right) & \geq 0 & \forall \mathbf{s}_{i} \\
f_{i s p}\left(\mathbf{s}_{i}\right) & \leq F_{I S P}\left(\mathbf{s}_{i}\right) & \forall \mathbf{s}_{i}
\end{array}
$$

In this LP we want to maximize the outgoing flow from all mesh gateway nodes given a minimum fairness constraints $\lambda_{0}$, which the same as maximizing the network throughput given the same fairness constraints. The first condition defines the flow of node $u$ as the difference between the outgoing flows and the incoming flows of all external virtual edges $\widehat{e}$ of node $u$. The second condition incorporates the fairness constraint in the flow of node $u$. The third condition defines the flow at the border mesh gateways. The rest of conditions are the same as defined in the Maximize Fairness section III-A.

\section{Link Scheduling}

Our objective is to give each link $\mathbf{L} \in G$ a transmission schedule $\mathcal{S}(\mathbf{L})$, which is the list of time slots and the corresponding (possibly combined) channels such that the schedule is interference-free and the overall throughput of the network is maximized. Let $X_{\widehat{e}, t, \mathbf{f}_{a b}} \in\{0,1\}$ be the indicator variable which is 1 only when $\widehat{e}$ will transmit at time $t$ using channel $\mathbf{f}_{a b}$.

We will focus on periodic schedules in this paper. A schedule is periodic with period $T$ if, for every virtual link $\widehat{e}$, every channel $\mathbf{f}_{a b}$, and time slot $t, X_{\widehat{e}, t, \mathbf{f}_{a b}}=X_{\widehat{e}, t+i \cdot T, \mathbf{f}_{a b}}$ for any integer $i>0$. For a virtual link $\widehat{e}$, let $\mathbf{I}(\widehat{e})$ denote the set of links $\widehat{e}^{\prime}$ that will cause interference if $\widehat{e}$ and $\widehat{e}^{\prime}$ are scheduled at the same time slot $t$ using the same channel $\mathbf{f}_{a b}$. Notice that a virtual edge $\widehat{e}^{\prime} \in \mathbf{I}(\widehat{e})$ if $\widehat{e}^{\prime}$ and $\widehat{e}$ share a common virtual node since any radio can only be active for either transmitting or receiving (but not both) at one specific channel. A schedule $\mathcal{S}$ is interference-free if $X_{\widehat{e}, t, \mathbf{f}_{a b}}+X_{\widehat{e}^{\prime}, t, \mathbf{f}_{p q}} \leq 1$ for any $\widehat{e}^{\prime} \in \mathbf{I}(\widehat{e})$, any time slot $t$, any channel $\mathbf{f}_{a b}$ and any $\mathbf{f}_{p q}$ with $\mathbf{I}_{\text {set }}\left(\mathbf{f}_{a b}, \mathbf{f}_{p q}\right)=1$. Conversely, we say $\left(\widehat{e}^{\prime}, \mathbf{f}_{p q}\right) \in$ $\mathbf{I}\left(\widehat{e}, \mathbf{f}_{a b}\right)$ if we cannot set $X_{\widehat{e}, t, \mathbf{f}_{a b}}=1$ and $X_{\widehat{e}^{\prime}, t, \mathbf{f}_{p q}}=1$ simultaneously for some time $t$.

We then mathematically formulate the necessary and sufficient condition for schedulable flow $f(e)=$ $\sum_{i, j} \sum_{\mathbf{f}_{a b} \in \mathbb{F}(e)} \alpha\left(\widehat{e}_{i, j}, \mathbf{f}_{a b}\right) \cdot \mathbf{c}\left(\widehat{e}_{i, j}, \mathbf{f}_{a b}\right)$. A flow $f$ (equivalently, whether a given vector $\alpha\left(\widehat{e}_{i, j}, \mathbf{f}_{a b}\right)$ for all virtual edge $\widehat{e}$ and $\mathbf{f}_{a b}$ is schedulable) is schedulable if and only if we can find solution $X_{\widehat{e}, t, \mathbf{f}_{a b}} \in\{0,1\}$ satisfying the following conditions.

$$
\left\{\begin{aligned}
X_{\widehat{e}, t, \mathbf{f}_{a b}}+X_{\widehat{e}^{\prime}, t, \mathbf{f}_{p q}} \leq 1 & \forall\left(\widehat{e}, \mathbf{f}_{p q}\right) \in \mathbf{I}\left(\widehat{e}, \mathbf{f}_{a b}\right), \\
& \forall \widehat{e}, t, \mathbf{f}_{a b} \\
\frac{\sum_{1 \leq t \leq T} X_{\widehat{e}, t, \mathbf{f}_{a b}}}{T}=\alpha\left(\widehat{e}, \mathbf{f}_{a b}\right) & \forall \widehat{e}, \forall \mathbf{f}_{a b} \\
\sum_{\widehat{e}: u \in \widehat{e}, \mathbf{f}_{a b} \in \mathbb{F}} X_{\widehat{e}, t, \mathbf{f}_{a b}} \leq \mathcal{I}(u) & \forall u, \forall t \in[1, T] \\
X_{\widehat{e}, t, \mathbf{f}_{a b}} \leq \delta\left(\widehat{e}, \mathbf{f}_{a b}\right) & \forall \widehat{e}, \forall \mathbf{f}_{a b} \\
X_{\widehat{e}, t, \mathbf{f}_{a b}} \in\{0,1\} & \forall \widehat{e}, \forall t, \forall \mathbf{f}_{a b}
\end{aligned}\right.
$$

The first condition says that a schedule should be interference-free. The second condition says the schedule should achieve the required flow $\alpha\left(\widehat{e}, \mathbf{f}_{a b}\right)$. The third condition specifies that the number of active links (incident on a node $u$ ) using all channels should be at most the number of radios that node $u$ has. Notice that node $u$ can be either the sender or the receiver for at most $\mathcal{I}(u)$ links simultaneously. The fourth condition says that a node can only use the channels that are available and operative by its radios. Observe that the virtual edges using a same radio of a wireless device $u$ will always interfere, i.e., $\widehat{e}_{i, k} \in \mathbf{I}\left(\widehat{e}_{i, j}\right)$ and $\widehat{e}_{k, j} \in \mathbf{I}\left(\widehat{e}_{i, j}\right)$ for any $k$.

1) Dynamic Channel Assignment: We then formulate the channel assignment that can facilitate the link scheduling and also schedulable flows. Dynamic channel assignment (DCA) methods will be studied in this paper where we assume that 
every wireless interface can dynamically change the channel (e.g., spectrum or CDMA code) for transmitting signals based on certain schedule.

Notice that in the link scheduling formulation, we use an indicator variable $X_{\widehat{e}, t, \mathbf{f}_{a b}}$ to denote whether virtual link $\widehat{e}$ will be active using a combined channel $\mathbf{f}_{a b}$ at time slot $t$. This indicator variable can also be directly interpreted as the dynamic channel assignment for nodes: if $X_{\widehat{e}, t, \mathbf{f}_{a b}}=1$ for $\widehat{e}=(u, v)$, then node $u$ will be assigned the channel $\mathbf{f}_{a b}$ to communicate with node $v$ at time slot $t$, and node $v$ will be assigned this channel $\mathbf{f}_{a b}$ for receiving signal. In other words, a feasible scheduling $X_{\widehat{e}, t, \mathbf{f}_{a b}}$ for all links, all combinedchannels, and all time slots already equivalently defines a feasible dynamic channel assignment. Thus, there is not any additional constraints when dynamic channel assignment is used.

2) Polynomial-time Schedulable Flows: It is widely known that it is NP-hard to decide whether a feasible scheduling $X_{e, t, \mathbf{f}_{a b}}$ exists when given the flow $f(e)$ (or equivalently, $\left.\alpha\left(e, \mathbf{f}_{a b}\right)\right)$ for wireless networks with interference constraints. For some interference models several papers gave relaxed necessary conditions and relaxed sufficient conditions for schedulable flows that can be decided in polynomial time. For example, without single channel network, for RTS/CTS model with uniform transmission range $R_{T}\left(v_{i}\right)$ and uniform interference range $R_{I}\left(v_{i}\right)$, [2] gave a sufficient condition $\alpha(e, \mathbf{f})+\sum_{e^{\prime} \in \mathbf{I}(e)} \alpha\left(e^{\prime}, \mathbf{f}\right) \leq 1$ and a necessary condition $\alpha(e, \mathbf{f})+\sum_{e^{\prime} \in \mathbf{I}(e)} \alpha\left(e^{\prime}, \mathbf{f}\right) \leq C(q)$. Here $C(q)$ is a constant depending on uniform $q=\frac{R_{I}(u)}{R_{T}(u)}$.

Definition 1: The set $\mathbf{I}\left(\widehat{e}, \mathbf{f}_{a b}\right)$ denotes the set of all pairs $\left(\widehat{e}^{\prime}, \mathbf{f}_{p q}\right)$ such that if virtual link $\widehat{e}$ will use channel $\mathbf{f}_{a b}$ for communication (at time slot $t$ ) and virtual link $\hat{e}^{t}$ will use channel $\mathbf{f}_{p q}$ for communication (at time slot $t$ ), then there will result in interference at some end-nodes of these links.

Notice that for any virtual edge $\widehat{e}^{\prime}=\left(\widehat{u_{i}}, \widehat{v_{j}}\right)$, and any $\mathbf{f}_{p q}$, we have $\left(\widehat{e}^{\prime}, \mathbf{f}_{p q}\right) \in \mathbf{I}\left(\widehat{e}, \mathbf{f}_{a b}\right)$ if $\widehat{e}^{\prime}$ is adjacent to either $\widehat{u_{i}}$ or $\widehat{v_{j}}$ since any radio of a node can only perform one operation (either one transmitting or one receiving, but not both). The only other scenario that $\left(\widehat{e}^{\prime}, \mathbf{f}_{p q}\right) \in \mathbf{I}\left(\widehat{e}, \mathbf{f}_{a b}\right)$ (when $\widehat{e}^{t}$ and $\widehat{e}$ do not share a common virtual node) is $\mathbf{I}_{s e t}\left(\mathbf{f}_{p q}, \mathbf{f}_{a b}\right)=1$ and $\widehat{e}^{\prime} \in \mathbf{I}(\widehat{e})$.

For an interference model $\mathcal{M}, \mathbf{I}_{\mathcal{M}}(e) \subseteq \mathbf{I}(e)$ will be defined based on the interference model $\mathcal{M}$ for the purpose of link scheduling. Take the PrIM model for example, $\mathbf{I}_{\mathcal{M}}(\widehat{e})$ is the set of virtual edges in $\mathbf{I}(\widehat{e})$ whose Euclidean length is at least that of $\widehat{e}$. We also define $\mathbf{I}_{\mathcal{M}}\left(\widehat{e}, \mathbf{f}_{a b}\right)$ as a subset of $\mathbf{I}\left(\widehat{e}, \mathbf{f}_{a b}\right)$ satisfying a special property depending on the interference model $\mathcal{M}$, i.e.,

$\mathbf{I}_{\mathcal{M}}\left(\widehat{e}, \mathbf{f}_{a b}\right)=\left\{\left(\widehat{e}, \mathbf{f}_{p q}\right) \mid\left(\widehat{e}^{\prime}, \mathbf{f}_{p q}\right) \in \mathbf{I}\left(\widehat{e}, \mathbf{f}_{a b}\right)\right.$ and $\left.\widehat{e}^{\prime} \in \mathbf{I}_{\mathcal{M}}(\widehat{e})\right\}$.

The required property will be explained later. For each of the interference models discussed in this paper, we later will present a necessary and a sufficient condition for schedulable flows (the proof of Theorem 1 is deferred to later section).

Theorem 1: Consider the active fraction $\alpha\left(\widehat{e}, \mathbf{f}_{a b}\right) \in[0,1]$ of each virtual link using each channel. A sufficient condition that this $\alpha$ is schedulable is,

$$
\alpha\left(\widehat{e}, \mathbf{f}_{a b}\right)+\sum_{\left(\widehat{e}^{\prime}, \mathbf{f}_{p q}\right) \in \mathbf{I}_{\mathcal{M}}\left(\widehat{e}, \mathbf{f}_{a b}\right)} \alpha\left(\widehat{e}, \mathbf{f}_{p q}\right) \leq 1, \forall \widehat{e}, \forall \mathbf{f}_{a b}
$$

A necessary condition that this $\alpha$ is schedulable is,

$$
\alpha\left(\widehat{e}, \mathbf{f}_{a b}\right)+\sum_{\left(\widehat{e}^{\prime}, \mathbf{f}_{p q}\right) \in \mathbf{I}_{\mathcal{M}}\left(\widehat{e}, \mathbf{f}_{a b}\right)} \alpha\left(\widehat{e}^{\prime}, \mathbf{f}_{p q}\right) \leq C_{\mathcal{M}}, \forall \widehat{e}, \forall \mathbf{f}_{a b}
$$

Here $C_{\mathcal{M}}$ is a constant depending on the specific interference model and $\gamma$. Notice that $\sum_{\widehat{e} \ni \widehat{u_{j}}, \mathbf{f}_{a b}} \alpha\left(\widehat{e}, \mathbf{f}_{a b}\right) \leq 1$ is always required for any virtual node $\widehat{u_{j}}$.

Thus, given a constant integer $C \in\left[1, C_{\mathcal{M}}\right]$, we will replace the condition $X_{\widehat{e}, t, \mathbf{f}_{a b}}+X_{\widehat{e}^{\prime}, t, \mathbf{f}_{p q}} \leq 1, \forall \widehat{e}, \forall \widehat{e}^{\prime}, \forall \mathbf{f}_{a b}, \mathbf{f}_{p q}$ such that $\left(\widehat{e}^{\prime}, \mathbf{f}_{p q}\right) \in \mathbf{I}\left(\widehat{e}, \mathbf{f}_{a b}\right)$ of feasible link scheduling with the following condition

$$
\alpha\left(\widehat{e}, \mathbf{f}_{a b}\right)+\sum_{\left(\widehat{e},, \mathbf{f}_{p q}\right) \in \mathbf{I}_{\mathcal{M}}\left(\widehat{e}, \mathbf{f}_{a b}\right)} \alpha\left(\widehat{e}^{\prime}, \mathbf{f}_{p q}\right) \leq C, \forall \widehat{e}, \forall \mathbf{f}_{a b}
$$

Notice that when $C=1$, we will show that the flow $f$ is guaranteed to have a feasible interference-free link scheduling. For any flow $f$ that can be implemented by an interferencefree link scheduling, we also have $C \leq C_{\mathcal{M}}$.

\section{Integrated Cross-Layer Mixed IP}

We then integrate all the conditions required into cross-layer mixed Integer Programming formulations which maximize the fairness among flows, or maximize the total throughout in the network. We summarize the mixed Integer Programming formulation for the joint routing, link-channel scheduling for multi-radio multi-channel multi-hop wireless networks as follows:

$$
\begin{array}{rlll}
\text { Mixed IP Flow Fairness: } & \max \lambda \\
\sum_{e \in \mathbf{E}^{+}(u)} f(e)-\sum_{e \in \mathbf{E}^{-}(u)} f(e) & \geq \lambda \ell(u) & \forall u \in V \\
\sum_{\mathbf{f}_{a b} \in \mathbb{R} c c(\widehat{e})} \alpha\left(\widehat{e}, \mathbf{f}_{a b}\right) \cdot \mathbf{c}\left(\widehat{e}, \mathbf{f}_{a b}\right) & =f(\widehat{e}) & \forall \widehat{e} \\
f(\widehat{e}) & \geq 0 & \forall \widehat{e} \\
\sum_{\widehat{e} \in e} f(\widehat{e}) & =f(e) & \forall e \\
\alpha\left(\widehat{e}, \mathbf{f}_{a b}\right) & \geq 0 & \forall \widehat{e} \\
\sum_{\widehat{e} \in e} \alpha\left(\widehat{e}, \mathbf{f}_{a b}\right) & \leq 1 & \forall e, \forall \mathbf{f}_{a b} \\
\sum_{\mathbf{f}_{a b}, \widehat{e}: \widehat{u}_{i} \in \widehat{e}} \alpha\left(\widehat{e}, \mathbf{f}_{a b}\right) & \leq 1 & \forall \widehat{u}_{i} \\
\sum_{\widehat{e} \in e} \alpha\left(\widehat{e}, \mathbf{f}_{a b}\right) & =\alpha\left(e, \mathbf{f}_{a b}\right) & \forall e \\
X_{\widehat{e}, t, \mathbf{f}_{a b}}+X_{\widehat{e}^{\prime}, t, \mathbf{f}_{p q}} & \leq 1 & \forall\left(\widehat{e}^{\prime}, \mathbf{f}_{p q}\right) \in \mathbf{I}\left(\widehat{e}, \mathbf{f}_{a b}\right), \\
& & \forall \widehat{e}, t, \mathbf{f}_{a b} \\
\sum_{1 \leq t \leq T} X_{\widehat{e}, t, \mathbf{f}_{a b}} & =\alpha\left(\widehat{e}, \mathbf{f}_{a b}\right) & \forall \widehat{e}, \forall \mathbf{f}_{a b} \\
\sum_{\widehat{e}: u \in \widehat{e} ; \mathbf{f}_{a b} \in \mathbb{F}} X_{\widehat{e}, t, \mathbf{f}_{a b}} & \leq \mathcal{I}(u) & \forall u, \forall t \in[1, T] \\
X_{\widehat{e}, t, \mathbf{f}_{a b}} & \leq \delta\left(\widehat{e}, \mathbf{f}_{a b}\right) & \forall \widehat{e}, \forall \mathbf{f}_{a b} \\
X_{\widehat{e}, t, \mathbf{f}_{a b}} & \in\{0,1\} & \forall \widehat{e}, \forall t, \forall \mathbf{f}_{a b}
\end{array}
$$

Recall that here $\sum_{\widehat{e} \in e} f(\widehat{e})=f(e), \forall e \in G$ is the total flow assigned to link $e$. Here, for a link $e=(u, v)$, a virtual edge $\widehat{e} \in e$ if $\widehat{e}$ connects a virtual node $\widehat{u_{i}}$ and a virtual node $\widehat{v_{j}}$. Observe that in the above mixed Integer Programming formulation, we consider both external virtual edges and internal virtual edges. Remember that $\widehat{\mathbf{E}}^{+}(u)$ (and $\widehat{\mathbf{E}}^{-}(u)$ ) contains all virtual (external and internal) edges incident at the group of virtual nodes by actual device $u$. Assigned flow $f(\widehat{e})$ is defined for all virtual edges; $\alpha\left(\widehat{e}, \mathbf{f}_{a b}\right)$ is only defined for external virtual edges. The solution of the internal virtual edge tells us about the radio switching and channel switching in a node. Although solving the above mixed Integer Programming will give us the optimum routing and link-channel scheduling, it is generally time-expensive to solve this since the original 
problem is NP-hard. Then we relax it to a linear programming by getting rid of the scheduling variables $X$. Based on previous study, we generally require that, given a constant integer $C \in$ $\left[1, C_{\mathcal{M}}\right]$, we need to solve the following Linear Programming (LP-Flow-fairness) (with polynomial number of variables and constraints) for $\alpha\left(\widehat{e}, \mathbf{f}_{a b}\right)$ :

$$
\begin{gathered}
\text { LP Flow fairness: } \max \lambda \\
\qquad \begin{array}{rll}
\sum_{e \in \mathbf{E}^{+}(u)} f(e)-\sum_{e \in \mathbf{E}^{-}(u)} f(e) & =f(u) & \forall u \in V \\
f(u) & \geq \lambda \ell(u) & \forall u \in V \\
\sum_{\mathbf{f}_{a b} \in \mathbb{R} c c(\widehat{e})} \alpha\left(\widehat{e}, \mathbf{f}_{a b}\right) \cdot \mathbf{c}\left(\widehat{e}, \mathbf{f}_{a b}\right) & =f(\widehat{e}) & \forall \widehat{e} \\
\sum_{\widehat{e} \in e} f(\widehat{e}) & =f(e) & \forall e \\
f(\widehat{e}) & \geq 0 & \forall \widehat{e} \\
\alpha\left(\widehat{e}, \mathbf{f}_{a b}\right) & \geq 0 & \forall \widehat{e} \\
\sum_{\widehat{e} \in e} \alpha\left(\widehat{e}, \mathbf{f}_{a b}\right) & \leq 1 & \forall e \\
\sum_{\mathbf{f}_{a b}, \widehat{e}: \widehat{u}_{i} \in \widehat{e}} \alpha\left(\widehat{e}, \mathbf{f}_{a b}\right) & \leq 1 & \forall \widehat{u}_{i} \\
\alpha\left(\widehat{e}, \mathbf{f}_{a b}\right)+\sum_{\left(\widehat{e}^{\prime}, \mathbf{f}_{p q}\right) \in \mathbf{I}_{\mathcal{M}}\left(\widehat{e}, \mathbf{f}_{a b}\right)} \alpha\left(\widehat{e}^{\prime}, \mathbf{f}_{p q}\right) & \leq C & \forall \widehat{e}, \forall \mathbf{f}_{a b} \\
\alpha\left(\widehat{e}, \mathbf{f}_{a b}\right) & \leq \delta\left(\widehat{e}, \mathbf{f}_{a b}\right) & \forall \widehat{e}, \forall \mathbf{f}_{a b}
\end{array}
\end{gathered}
$$

Notice condition $\sum_{\mathbf{f}_{a b}, \widehat{e}: u \in \widehat{e}} \alpha\left(\widehat{e}, \mathbf{f}_{a b}\right) \leq \mathcal{I}(u)$, for any node $u$, is already implied by the condition $\sum_{\mathbf{f}_{a b}, \widehat{e}: \widehat{u}_{i} \in \widehat{e}} \alpha\left(\widehat{e}, \mathbf{f}_{a b}\right) \leq$ $1 \forall \widehat{u_{i}}$ Similarly, we can formulate a linear programming for LP-Flow-throughput such that the solution $\alpha\left(\widehat{e}, \mathbf{f}_{a b}\right)$ is guaranteed to have a feasible link and channel scheduling: the above second condition is changed to $f(u) \geq \lambda_{0} \ell(u)$ for a given $\lambda_{0} \geq 0$, and the objective function becomes $\max \sum_{u \in V} f(u)$. The rest of the paper is devoted to design a polynomial time method that can find such link and channel scheduling that satisfies the solution $\alpha\left(\widehat{e}, \mathbf{f}_{a b}\right)$ from the LP. We will also prove that the achieved fairness or throughput is within a constant factor of the optimum.

\section{EFFICIENT TDMA SCHEDULING}

In this section, we present an efficient algorithm to find a feasible link scheduling given a flow found by our LP.

\section{A. Polynomial Time Scheduling Method}

We will first present a centralized scheduling for link transmission. Our method is based on some algorithms presented in [27] on link scheduling for networks with single channel. However, the key difference is we assume multiple-channels and multiple-radios per node. Assume $T$ is the number of time slots per scheduling period. We need to schedule $T \cdot \alpha\left(\widehat{e}, \mathbf{f}_{a b}\right)$ time-slots for a virtual link $\widehat{e}$ using channel $\mathbf{f}_{a b}$. Notice that here we only need to schedule the transmission of external virtual edges: when to transmit and which combined-channel to use. For simplicity, we assume that the choice of $T$ leads $T \cdot \alpha\left(\widehat{e}, \mathbf{f}_{a b}\right)$ to be integer for every virtual edge $\widehat{e}$ and $\mathbf{f}_{a b}$. In addition, we need to ensure that each scheduled pair (link, channel) is interference-free, satisfies the radio and channelavailability constraints of all nodes. Algorithm 1 illustrates our scheduling method. The basic idea is to first sort the external virtual links based on some specific order and then process the requirement $\alpha\left(\widehat{e}, \mathbf{f}_{a b}\right)$ for each of the possible channel $\mathbf{f}_{a b}$. Assume that there is a table $\mathbf{Y}(t)$ for each virtual node $\widehat{u_{j}}$, i.e. the $j$ th radio at node $u$. The table stores the current assignment for (virtualEdge, channel) pair, i.e., an entry in $\mathbf{Y}(t),\left(\widehat{e}, \mathbf{f}_{a b}\right)$, means that node $u$ will use its $j$ th NIC to transmit at time $t$ using channel $\mathbf{f}_{a b}$ for link $\widehat{e}$, if the directed virtual edge $\widehat{e}$ start from virtual node $\widehat{u_{j}}$; otherwise, node $u$ will use NIC $j$ to receive at time $t$ using channel $\mathbf{f}_{a b}$ for link $\widehat{e}$ ended at virtual node $\widehat{u_{j}}$.

For a virtual edge $\widehat{e}_{i}$, we will need to find $N\left(\widehat{e}_{i}, \mathbf{f}_{a b}\right)=$ $T \cdot \alpha\left(\widehat{e}_{i}, \mathbf{f}_{a b}\right)$ empty entries that will not cause interference to other scheduled pairs of (link, channel). If there are available consecutive time slots of a radio, we will choose consecutive time slots (to reduce the channel switching cost).

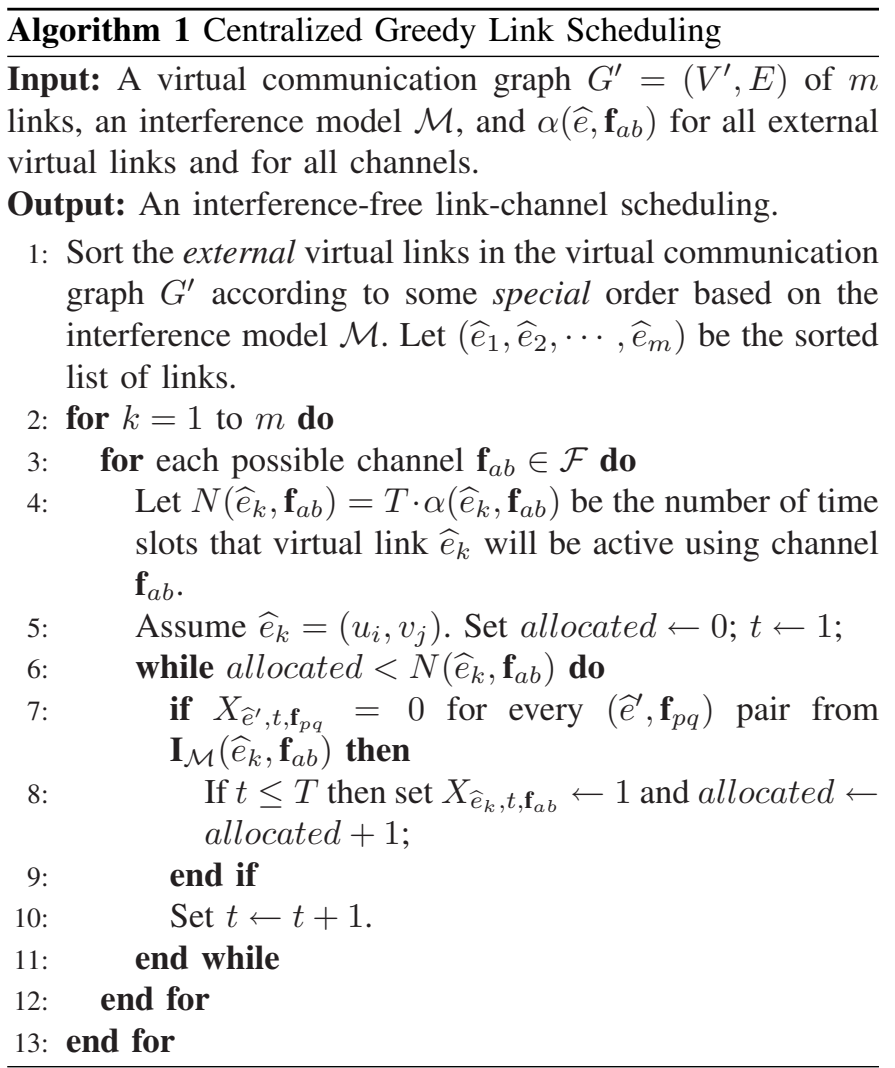

Notice that our algorithm relies on some special sorting of the links, which depends on the interference models. We are proposing different sorting algorithms for the different interference models because the models are different: some based on distance between transmitting nodes, some based on distance between transmitting and receiving nodes, and others are based on the interference range of the transmitting node only; we could not find one common sorting that works for all interference models. We process links in some special sorted order. When processing the $i^{t h}$ virtual link $\widehat{e}_{i}$, we process the channels in order and assign link $\widehat{e}_{i}$ the earliest (not need to be consecutive) $N\left(\widehat{e}_{i}, \mathbf{f}_{a b}\right)=T \cdot \alpha\left(\widehat{e}_{i}, \mathbf{f}_{a b}\right)$ time slots using channel $\mathbf{f}_{a b}$ which will not cause any interference to scheduled links, and satisfy the radio and channel-availability constraints. It is worth noting the time complexity of Algorithm 1 is $O(m \log m)$, most of the time is spent in sorting the links, the inner nested loops in steps 3 to 12 have a constant upper bound of $\left(\frac{K(K+1)}{2}\right)^{2} \times T$, the over all time complexity is $O(m \log m)+O\left(m\left(\frac{K(K+1)}{2}\right)^{2} T\right)=O(m \log m)$ for sufficiently large $m$. Notice that $\alpha$ can be found in time $O\left(\mathrm{~m}^{3.5}\right)$ since a LP of $m$ variables can be solved in time $O\left(\mathrm{~m}^{3.5}\right)$ by using interior point method.

We then explain in detail how to sort the links for different 
interference models and also define the $\mathbf{I}_{\mathcal{M}}(\widehat{e})$ for a link $\widehat{e}$ with respect to all interference models $\mathcal{M}$ (namely, RTS/CTS model, fPrIM model, PrIM model, TxIM model).

RTS/CTS: One order for RTS/CTS model is to sort all links in the decreasing order of their interference radius as follows: pick a link from $G^{\prime}$ 's conflict graph $\mathbb{G}$, say $l$, that has the smallest number of interfered links in the remaining $\mathbb{G}$ graph and then remove link $l$ from $\mathbb{G}$; repeat this till the graph $\mathbb{G}$ is empty; then the links (in the original graph) are sorted by their reverse removal order. For the performance proof, we will adopt this sorting. Here the interference radius of a link $e=\left(v_{i}, v_{j}\right)$ is defined as $r_{i, j}=\max \left\{R_{I}\left(v_{i}\right), R_{I}\left(v_{j}\right)\right\}$. The interference radius of a virtual edge $\widehat{e}_{i, j}$ (with actual end nodes $u$ and $v$ ) is same as that of the actual link $e=(u, v)$. The set $I_{R T S / C T S}(\widehat{e})$ is the set of links whose interference radius is at least that of $\widehat{e}$ and interfering with $\widehat{e}$ under RTS/CTS model. In this case, the constant $C_{\mathcal{M}} \leq 120$, proof is similar to Lemma 3 of [27].

fPrIM: In fPrIM model, we again consider the (virtual) conflict graph. We choose the vertex, which is the virtual link in the virtual communication graph, with the largest value $d_{i, j}^{i n}-d_{i, j}^{\text {out }}$ in the residue conflict graph; remove the vertex and its incident edges. Where $d_{i, j}^{i n}$ and $d_{i, j}^{\text {out }}$ are the in-degree and out-degree of vertex $\mathbf{L}_{i, j}$ in the conflict graph for the fPrIM interference model. Repeat this process until there is no vertex in the graph. Then the links (in the original graph) are sorted by their reverse removal order. The set $I_{f \operatorname{PrIM}}(\widehat{e})$ is the set of incoming links of $\widehat{e}$ that interfere $\widehat{e}$. Here a virtual link $e^{\prime}$ is called the incoming link of $\widehat{e}$ if the activation of link $e^{\prime}$ will cause the interference at the receiving node of link $\widehat{e}$, i.e., the vertex corresponding to $e^{\prime}$ in the conflict graph $F_{G}^{F P}$ is the incoming-neighbor of the vertex for $\widehat{e}$ in the graph $F_{G}^{F P}$. In this case, $C_{\mathcal{M}} \leq\left\lceil\frac{2 \pi}{\arcsin \frac{\gamma-1}{2 \gamma}}\right\rceil$, e.g., $C_{\mathcal{M}} \leq 25$ when $\gamma=2$, proof is similar to Lemma 6 of [27]).

TxIM: Recall in TxIM that transmit power is not fixed and the transmission of node $v_{i}$ is successful if and only if for any other transmission $v_{k}$ the distance between $v_{i}$ and $v_{k}$ is more than the sum of the transmission range of $v_{i}$ and $v_{k}$. In TxIM model, we sort the links according to the interference radius of the sending node in a non-increasing order by following the same method in RTS/CTS except we process the links in the order they were picked. Notice that links in this paper are always directed. For a link $e=\left(v_{i}, v_{j}\right)$, the set $I_{T x I M}(\widehat{e})$ is the set of virtual links $e^{\prime}=\left(v_{p}, v_{q}\right)$ such that the interference range $R_{I}\left(v_{p}\right)$ of node $v_{p}$ is at least the interference range $R_{I}\left(v_{i}\right)$ of node $v_{i}$ and $\left\|v_{p}-v_{i}\right\| \leq R_{I}\left(v_{p}\right)+R_{I}\left(v_{i}\right)$, i.e., nodes $v_{p}$ and $v_{i}$ interfere with each other. In this case, the constant $C_{\mathcal{M}} \leq 5$, proof is similar to claim 2 of [13].

PrIM: In PrIM model, we sort links according to their Euclidean length in a non-increasing order by following the same method in RTS/CTS except we process the links in the order they were picked; recall that in PrIM when the Euclidean distance between source and destination nodes is smaller, the interference is less. $\operatorname{IPrIM}_{\operatorname{Pr}}(\widehat{e})$ is the set of links whose Euclidean distance is at least that of $\widehat{e}$. In this case, the constant $C_{\mathcal{M}} \leq\left(5+\frac{4}{\eta}\right)^{2}$, proof is similar to claim 2 of [13].

\section{B. Correctness and Performance Guarantee}

We first show that Algorithm 1 indeed finds an interferencefree scheduling when $\alpha\left(\widehat{e}, \mathbf{f}_{a b}\right)$ is a feasible solution of linear programming LP-Flow-throughput and LP-Flow-fairness where the adjustable constant $C$ is set as 1 .

Theorem 2: Algorithm 1 produces a feasible interferencefree link-channel scheduling when $\alpha\left(\widehat{e}, \mathbf{f}_{a b}\right)$ is a feasible solution of LP using $C=1$.

Proof: From the Linear Program LP-Flow-fairness for $\alpha\left(\widehat{e}, \mathbf{f}_{a b}\right)$, which is applicable to each interference model mentioned above, we get the solution $\alpha$. Essentially we need to show that Algorithm 1 will terminate. Notice that after the algorithm terminates, we know that for every link $\widehat{e}$ and every channel $\mathbf{f}_{a b}$, it is already assigned a fraction $\alpha\left(\widehat{e}, \mathbf{f}_{a b}\right)$ time slot in a schedule period $T$. Consider a specific link $\widehat{e}$ that is to be processed. Based on the special sorting used by our algorithm for each interference model, we know that all virtual links $\widehat{e}^{\prime}$ that have been processed and conflict with $\widehat{e}$ (interfering $\widehat{e}$ or being interfered by link $\widehat{e}$ ) must be a subset of $\mathbf{I}_{\mathcal{M}}(\widehat{e})$. Notice that it may be not exactly $\mathbf{I}_{\mathcal{M}}(\widehat{e})$ due to possible different tie-breaking of sorting. Recall that in our linear programming, we had a condition that, for every channel $\mathbf{f}_{a b}, \alpha\left(\widehat{e}, \mathbf{f}_{a b}\right)+\sum_{\left(\widehat{e}^{\prime}, \mathbf{f}_{p q}\right) \in \mathbf{I}_{\mathcal{M}}\left(\widehat{e}, \mathbf{f}_{a b}\right)} \alpha\left(\widehat{e}^{\prime}, \mathbf{f}_{a b}\right) \leq 1$. This implies that, for each $\mathbf{f}_{a b}$,

$$
N\left(\widehat{e}, \mathbf{f}_{a b}\right)+\sum_{\left(\widehat{e}^{\prime}, \mathbf{f}_{p q}\right) \in \mathbf{I}_{\mathcal{M}}\left(\widehat{e}, \mathbf{f}_{a b}\right)} N\left(\widehat{e}^{\prime}, \mathbf{f}_{p q}\right) \leq T, \forall \widehat{e} .
$$

Thus, we can always find $N\left(\widehat{e}, \mathbf{f}_{a b}\right)=T \cdot \alpha\left(\widehat{e}, \mathbf{f}_{a b}\right)$ time-slots among $T$ slots in a period for link $\widehat{e}$ using channel $\mathbf{f}_{a b}$, since all conflict links that already have been processed by Algorithm 1 occupy at most $\sum_{\left(\widehat{e}^{\prime}, \mathbf{f}_{p q}\right) \in \mathbf{I}_{\mathcal{M}}\left(\widehat{e}, \mathbf{f}_{a b}\right)} N\left(\widehat{e}^{\prime}, \mathbf{f}_{p q}\right) \leq T-N\left(\widehat{e}, \mathbf{f}_{a b}\right)$ time slots. Notice that each virtual node $\widehat{u}_{i}$ only has one radio. Since the total number of time slots needed for a virtual node $\widehat{u_{i}}$ is $\sum_{\widehat{e}: \widehat{u_{i}} \in \widehat{e}, \mathbf{f}_{a b}} T \cdot \alpha\left(\widehat{e}, \mathbf{f}_{a b}\right) \leq T$, among $T$ time slots, we can always find time slots for link $\widehat{e}$ using a channel $\mathbf{f}_{a b}$ (after considering all conflicting links scheduled before).

Since $\alpha\left(\widehat{e}, \mathbf{f}_{a b}\right)>0$ for the current virtual link $e=\left(\widehat{u_{i}}, \widehat{v_{j}}\right)$, we know that $\delta\left(u, \mathbf{f}_{a b}\right)=\delta\left(v, \mathbf{f}_{a b}\right)=1$ from $\delta\left(u, \mathbf{f}_{a b}\right) \geq$ $\alpha\left(\widehat{e}, \mathbf{f}_{a b}\right)$ and $\delta\left(v, \mathbf{f}_{a b}\right) \geq \alpha\left(\widehat{e}, \mathbf{f}_{a b}\right)$. In other words, both the end-nodes, $\widehat{u_{i}}$ and $\widehat{v_{j}}$, of the link $\widehat{e}$ can operate on channel $\mathbf{f}_{a b}$.

This finishes our proof.

Theorem 3: Algorithm 1, together with the linear programming formulation LP-Flow-fairness, produces a feasible interference-free link-channel scheduling whose achieved fairness is at least $\frac{1}{C_{\mathcal{M}}}$ of the optimum, when $\alpha\left(\widehat{e}, \mathbf{f}_{a b}\right)$ is a feasible solution of LP using $C=1$.

Proof: Consider an optimum flow assignment defined by $\alpha^{*}(\widehat{e}, \mathbf{f})$, i.e., the flow supported by a link $\widehat{e}$ is $\sum_{\mathbf{f}_{a b}} \alpha^{*}\left(\widehat{e}, \mathbf{f}_{a b}\right)$. $\mathbf{c}\left(\widehat{e}, \mathbf{f}_{a b}\right)$. From Theorem 1, we know that

$$
\alpha^{*}\left(\widehat{e}, \mathbf{f}_{a b}\right)+\sum_{\left(\widehat{e}^{\prime}, \mathbf{f}_{p q}\right) \in \mathbf{I}_{\mathcal{M}}\left(\widehat{e}, \mathbf{f}_{a b}\right)} \alpha^{*}\left(\widehat{e}^{\prime}, \mathbf{f}_{p q}\right) \leq C_{\mathcal{M}} .
$$

Define a new flow $\alpha^{\prime}$ as $\alpha^{\prime}\left(\widehat{e}, \mathbf{f}_{a b}\right)=\frac{\alpha^{*}\left(\widehat{e}, \mathbf{f}_{a b}\right)}{C_{\mathcal{M}}}$. Obviously,

$$
\alpha^{\prime}\left(\widehat{e}, \mathbf{f}_{a b}\right)+\sum_{\left(\widehat{e}^{\prime}, \mathbf{f}_{p q}\right) \in \mathbf{I}_{\mathcal{M}}\left(\widehat{e}, \mathbf{f}_{a b}\right)} \alpha^{\prime}\left(\widehat{e}^{\prime}, \mathbf{f}_{p q}\right) \leq 1 .
$$


It is easy to show that the new flow $\alpha^{\prime}$ satisfies all conditions of our linear programming LP-Flow-fairness. In other words, $\alpha^{\prime}$ is a feasible solution for this LP. Consequently, the solution of LP-Flow-fairness is at least that of $\alpha^{\prime}$, which is $\frac{1}{C_{\mathcal{M}}}$ of the optimum. This finishes the proof.

It is easy to show that our LP solution for LP-Flowthroughput also finds a flow assignment whose total flow is at least $\frac{1}{C_{\mathcal{M}}}$ of the optimum when $\lambda_{0}=0$. If we relax the condition $f(u) \geq \lambda_{0} \ell(u)$ to $f(u) \geq \frac{\lambda_{0}}{C_{\mathcal{M}}} \ell(u)$ in the linear programming LP-Flow-throughput, we can show that our linear programming will find a flow assignment whose total achieved throughput is at least $\frac{1}{C_{\mathcal{M}}}$ of the optimum and the achieved fairness is at least $\frac{\lambda_{0}}{C_{\mathcal{M}}}$ (instead of the required $\lambda_{0}$ ).

Theorem 4: Algorithm 1, together with the linear programming formulation LP-Flow-throughput, produces a feasible interference-free link-channel scheduling with throughput at least $\frac{1}{C_{\mathcal{M}}}$ of the optimum, and the fairness at least $\frac{\lambda_{0}}{C_{\mathcal{M}}}$, when $\alpha\left(\widehat{e}, \mathbf{f}_{a b}\right)$ is a feasible solution of LP using $C=1$.

The proof of Theorem 1: The rest of the subsection is devoted to the proof of Theorem 1, which is based on some theorems proved in [13], [27]. The sufficient condition comes from the correctness of Algorithm 1 which gives a valid link-channel schedule. We then show the correctness of that necessary condition. Similar to the Claim 2 of [13], we show that

Lemma 5: For interference models PrIM or TxIM, there exists a constant $C_{1}$ such that for any time slot $\tau$, any interference-free link scheduling $\mathcal{S}$ satisfies $X_{\widehat{e}, \tau, \mathbf{f}_{a b}}+$ $\sum_{\left(\widehat{e}^{\prime}, \mathbf{f}_{p q}\right) \in \mathbf{I}_{\mathcal{M}}\left(\widehat{e}, \mathbf{f}_{a b}\right)} X_{\widehat{e}^{\prime}, \tau, \mathbf{f}_{p q}} \leq C_{1}$.

Proof: Consider the interference model TxIM first and a virtual link $\widehat{e}=\left(u_{i}, v_{j}\right)$. We partition the region into 6 sectors centered at node $u$, each with angle $\pi / i$. We then show that there are at most 1 node in each sector from $\mathbf{I}_{\mathcal{M}}\left(\widehat{e}, \mathbf{f}_{a b}\right)$. Assume that there are two nodes $w$ and $w^{\prime}$ with $\|u-w\| \geq\left\|u-w^{\prime}\right\|$. Then it is easy to show that $\left\|w^{\prime}-u\right\| \leq\left\|w^{\prime}-w\right\|$. Notice that $u$ and $w$ conflicts implies $\|u-w\| \leq R_{T}(u)+R_{T}(w)$. Node $w^{\prime}$ is in $\mathbf{I}_{\mathcal{M}}\left(\widehat{e}, \mathbf{f}_{a b}\right)$ implies that $R_{T}\left(w^{\prime}\right) \geq R_{T}(u)$. Thus, $\left\|w^{\prime}-w\right\| \leq\left\|w^{\prime}-u\right\| \leq$ $\|u-w\| \leq R_{T}(u)+R_{T}(w) \leq R_{T}\left(w^{\prime}\right)+R_{T}(w)$. In other words, $w$ and $w^{\prime}$ conflicts. Consequently, we know that $\sum_{\left(\widehat{e}^{\prime}, \mathbf{f}_{p q}\right) \in \mathbf{I}_{\mathcal{M}}\left(\widehat{e}, \mathbf{f}_{a b}\right)} X_{\widehat{e}^{\prime}, \tau, \mathbf{f}_{p q}} \leq 5$ for TxIM interference model. The proof of PrIM model is little bit different and is omitted here due to space limit.

Similarly, we have (proofs are omitted)

Lemma 6: There exists a constant $C_{2}$ such that for any time slot $\tau$, any RTS/CTS interference-free link scheduling $\mathcal{S}$ must satisfy that $X_{\widehat{e}, \tau, \mathbf{f}_{a b}}+\sum_{\left(\widehat{e}^{\prime}, \mathbf{f}_{p q}\right) \in \mathbf{I}_{R T S / C T S}\left(\widehat{e}, \mathbf{f}_{a b}\right)} X_{\widehat{e}^{\prime}, \tau, \mathbf{f}_{p q}} \leq C_{2}$.

Lemma 7: For any time slot $\tau$, any interference-free link scheduling $\mathcal{S}$ under fPrIM interference model must satisfy that $X_{\widehat{e}, \tau, \mathbf{f}_{a b}}+\sum_{\left(\widehat{e}^{\prime}, \mathbf{f}_{p q}\right) \in \mathbf{I}_{f P r I M}\left(\widehat{e}, \mathbf{f}_{a b}\right)} X_{\widehat{e}^{\prime}, \tau, \mathbf{f}_{p q}} \leq\left\lceil\frac{2 \pi}{\arcsin \frac{\gamma-1}{2 \gamma}}\right\rceil$.

Consequently we can generally claim that any interferencefree scheduling should satisfy that

$$
X_{\widehat{e}, \tau, \mathbf{f}_{a b}}+\sum_{\left(\widehat{e}^{\prime}, \mathbf{f}_{p q}\right) \in \mathbf{I}_{\mathcal{M}}\left(\widehat{e}, \mathbf{f}_{a b}\right)} X_{\widehat{e}^{\prime}, \tau, \mathbf{f}_{a b}} \leq C_{\mathcal{M}}
$$

for a constant $C_{\mathcal{M}}$ depending on the interference model $\mathcal{M}$. Thus, $\sum_{t=1}^{T}\left(X_{\widehat{e}, \tau, \mathbf{f}_{a b}}+\sum_{\left(\widehat{e}^{\prime}, \mathbf{f}_{p q}\right) \in \mathbf{I}_{\mathcal{M}}\left(\widehat{e}, \mathbf{f}_{a b}\right)} X_{\widehat{e}^{\prime}, \tau, \mathbf{f}_{a b}}\right) / T \leq$ $C_{\mathcal{M}}$. Equivalently, for each channel $\mathbf{f}_{a b}$ and virtual edge $\widehat{e}$, $\alpha\left(\widehat{e}, \mathbf{f}_{a b}\right)+\sum_{\left(\widehat{e}^{\prime}, \mathbf{f}_{p q}\right) \in \mathbf{I}_{\mathcal{M}}\left(\widehat{e}, \mathbf{f}_{a b}\right)} \alpha\left(\widehat{e}^{\prime}, \mathbf{f}_{a b}\right) \leq C_{\mathcal{M}}$, which finishes the proof of Theorem 1 .

\section{Improvement and Implementations}

We can also use parametric searching to improve the overall achieved flow. Assume that we replace $\alpha\left(\widehat{e}, \mathbf{f}_{a b}\right)+\sum_{\left(\widehat{e}^{\prime}, \mathbf{f}_{p q}\right) \in \mathbf{I}_{\mathcal{M}}\left(\widehat{e}, \mathbf{f}_{a b}\right)} \alpha\left(\widehat{e}^{\prime}, \mathbf{f}_{p q}\right) \leq 1$, by $\alpha\left(\widehat{e}, \mathbf{f}_{a b}\right)+$ $\sum_{\left(\widehat{e}^{\prime}, \mathbf{f}_{p q}\right) \in \mathbf{I}_{\mathcal{M}}\left(\widehat{e}, \mathbf{f}_{a b}\right)} \alpha\left(\widehat{e}^{\prime}, \mathbf{f}_{p q}\right) \leq C$ for an integer $C>1$. The the computed flow $f$ may be schedulable or may be not schedulable. Recall that for any schedulable flow, we should have $\alpha\left(\widehat{e}, \mathbf{f}_{a b}\right)+\sum_{\left(\widehat{e}^{\prime}, \mathbf{f}_{p q}\right) \in \mathbf{I}_{\mathcal{M}}\left(\widehat{e}, \mathbf{f}_{a b}\right)} \alpha\left(\widehat{e}^{\prime}, \mathbf{f}\right) \leq C_{\mathcal{M}}$. In other words, it is sufficient to use integer $C \leq C_{\mathcal{M}}$ to find all schedulable flows.

We will solve the corresponding linear programming using an integer $C$ starting from $C_{\mathcal{M}}$ and find the $\alpha$. We then call our scheduling method (Algorithm 1) to find whether $\alpha$ is schedulable or not. If we cannot find a schedule for it, we then decrease $C$ by 1 and then repeat the above steps. Notice that the above steps can be repeated for at most $C_{\mathcal{M}}$ rounds. If we can find a valid schedule for an integer $C^{\prime} \in\left[1, C_{\mathcal{M}}\right]$, the found flow achieves fairness (or throughput) at least $\frac{C^{\prime}}{C_{\mathcal{M}}}$ times of the optimum.

Notice that the LP solution only shows the amount of traffics each link should support for each pair of source and destination nodes. Given this network flow assignment, it is not difficult to find paths connecting any given pair source and destination. A simple flow-splitting method works as follows. Let $f^{\prime}(e)=f(e)$. Given a flow for a pair source $s$ and destination $d$, finding the link with the smallest positive flow, say $f^{\prime}(e)$; then removing a path $\mathbf{P}$ (put it to $\Pi$ ) connecting $s$ and $d$ using link $e$; the load of path $\mathbf{P}$ is $f(\mathbf{P})=\min _{a \in \mathbf{P}} f^{\prime}(a)$; update the flow $f^{\prime}(a)$ for each link $a \in \mathbf{P}$ as $f^{\prime}(a)-f^{\prime}(e)$; repeating the above steps until all links $f^{\prime}$ are 0 . Obviously, the above procedure will stop after at most $m$ rounds since each round at least one additional link's load will become 0 . It implies that there are at most $m$ paths to connecting $s$ and $d$ for routing the network flow, each with a load $f(\mathbf{P})$. When routing the actual sessions, we can choose the path based on certain probability (a path $P \in \Pi$ is selected with probability $f(P) / f(u)$ where $f(u)$ is the achievable flow for node $u$ ) such that the expected total traffics routed through $\mathbf{P}$ is $f(\mathbf{P})$. Notice that it is NP-hard to decide whether we can assign traffics to paths such that the total traffics routed on a path is at most $f(\mathbf{P})$ since this problem can reduce to subset sum problem.

\section{Additional Constraints}

Observe that our previous LP based solution works for both unicast, data collection from all nodes to a set of sink nodes, and generally multicommodity flow where different types of applications like audio, video, text are serviced simultaneously by the network. Our linear programming based approach also works for any additional constraints that can be expressed as linear formula of $\alpha$.

One possible constraint is for resource constraint wireless networks. Assume that each node $u \in V$ in the network will spend $\phi\left(\left(u_{i}, v_{j}\right), \mathbf{f}_{a b}\right)$ resources (e.g., power) for relaying a unit amount of data using $i$ th NIC to a neighboring node $v$ 
who will receive at the $j$ th NIC using a channel $\mathbf{f}_{a b}$. Further, each node $u$ has a given amount of resources $\Phi(u)>0$. The objective is then to maximize the network throughput (or fairness) that can be achieved by such a network while the network will last for a certain duration $D$. Then we need an additional constraint for each such constraint resource

$\sum_{i, v_{j}, \mathbf{f}_{a b}} \phi\left(\left(u_{i}, v_{j}\right), \mathbf{f}_{a b}\right) \cdot D \cdot \mathbf{c}\left(\left(u_{i}, v_{j}\right), \mathbf{f}_{a b}\right) \cdot \alpha\left(\left(u_{i}, v_{j}\right), \mathbf{f}_{a b}\right) \leq \Phi(u)$.

Another possible constraint is to maximize a total multicommodity flow when each node will charge a certain cost for relaying while there is a total budget for routing multicommodity flow. Assume that, for each virtual edge $\widehat{e}=\left(u_{i}, v_{j}\right)$, node $u$ will charge $\mathbf{p}\left(\widehat{e}, \mathbf{f}_{a b}\right)$ for relaying a unit amount of data to a neighboring node $v$ using a channel $\mathbf{f}_{a b}$. Further assume that the total budget for the multicommodity flow is $B$. The objective is to maximize the total throughput within budget $B$. Then it is easy to show that the additional constraint for our LP formulation is that

$$
\sum_{u, v} \sum_{i, j, \mathbf{f}_{a b}} \mathbf{p}\left(\left(u_{i}, v_{j}\right), \mathbf{f}_{a b}\right) \cdot \mathbf{c}\left(\left(u_{i}, v_{j}\right), \mathbf{f}_{a b}\right) \cdot \alpha\left(\left(u_{i}, v_{j}\right), \mathbf{f}_{a b}\right) \leq B .
$$

We can show that if these extra constraints are added to the requirement, our algorithm still works and Theorems 3 and 4 still hold.

\section{E. Stochastic Link Capacity}

So far, as done in previous study of network capacity and throughput, we assumed that the capacity of the links in a mesh network is deterministic. However, this is practically not the case as links are often subject to environmental variations and failures. In such a case, effective data communication is a challenge. Assume that for each virtual link $\widehat{e}=\left(u_{i}, v_{j}\right)$, its capacity is randomly drawn from a certain range with a certain probability distribution. For simplicity, we assume that the link capacity is at least $\mathbf{c}(\widehat{e}, \mathbf{f}) / \beta_{1}$ with high probability and at most $\mathbf{c}(\widehat{e}, \mathbf{f}) \cdot \beta_{2}$ for some constants $\beta_{1}>1$ and $\beta_{2}>1$ where $\mathbf{c}(\widehat{e}, \mathbf{f})$ is the expected capacity using a (possible-combined) channel f. Then we need to find a flow assignment that will maximize the expected throughput and also the flow should be schedulable with high probability. Clearly the realization of the network flow depending on the realization of the link capacities. Our approach will be to use $\mathbf{c}(\widehat{e})$ as input of our algorithm to find $\alpha(\widehat{e}, \mathbf{f})$. The flow assignment $\alpha \cdot \mathbf{c}$ may be not schedulable for some realization of the capacity (the actual time-slots needed depending on the capacity realization). Since for any virtual link $\widehat{e}$, its realized capacity is at least $\mathbf{c}(\widehat{e}, \mathbf{f}) / \beta_{1}$, we show that

Lemma 8: The flow assignment $\alpha\left(\widehat{e}, \mathbf{f}_{a b}\right) \cdot \mathbf{c}\left(\widehat{e}, \mathbf{f}_{a b}\right) / \beta_{1}$ is schedulable with high probability.

Proof: Let $y\left(\widehat{e}, \mathbf{f}_{a b}\right)$ be an actual realization of link capacity. Then the time-slots needed by link $\widehat{e}$ on channel $\mathbf{f}_{a b}$ is $\theta\left(\widehat{e}, \mathbf{f}_{a b}\right)=\frac{\alpha\left(\widehat{e}, \mathbf{f}_{a b}\right) \cdot \mathbf{c}\left(\widehat{e}, \mathbf{f}_{a b}\right)}{\beta_{1} \cdot y\left(\widehat{e}, \mathbf{f}_{a b}\right)}$. Observe that, from our LP formulation, the computed $\alpha$ satisfies that $\alpha\left(\widehat{e}, \mathbf{f}_{a b}\right)+$ $\sum_{\left(\widehat{e}^{\prime}, \mathbf{f}_{p q}\right) \in \mathbf{I}_{\mathcal{M}}\left(\widehat{e}, \mathbf{f}_{a b}\right)} \alpha\left(\widehat{e}^{\prime}, \mathbf{f}_{p q}\right) \leq 1$. Notice, we essentially proved in 2 that Algorithm 1 can find a schedule if $\theta\left(\widehat{e}, \mathbf{f}_{a b}\right)+$ $\sum_{\left(\widehat{e}^{\prime}, \mathbf{f}_{p q}\right) \in \mathbf{I}_{\mathcal{M}}\left(\widehat{e}, \mathbf{f}_{a b}\right)} \theta\left(\widehat{e}^{\prime}, \mathbf{f}_{p q}\right) \leq 1$, which is satisfied with high probability since $\frac{\mathbf{c}\left(\widehat{e}, \mathbf{f}_{a b}\right)}{\beta_{1} \cdot y\left(\widehat{e}, \mathbf{f}_{a b}\right)} \leq 1$ with high probability. This finishes the proof.

Notice for any realization, $y\left(\widehat{e}, \mathbf{f}_{a b}\right) \leq \beta_{2} \mathbf{c}\left(\widehat{e}, \mathbf{f}_{a b}\right)$ with high probability implies that, the maximum flow achieved by any network realization is at most $\beta_{2}$ times of the maximum flow computed by our LP formulation. Consequently, the flow achieved based on $\frac{\alpha \cdot \mathbf{c}}{\beta_{1}}$ is at least $\frac{1}{C_{\mathcal{M}} \cdot \beta_{1} \cdot \beta_{2}}$ fraction of the maximum flow achieved.

\section{Performance Evaluation}

In this section, we will exam the fairness and maximal throughput in wireless mesh networks with various channels, radios, and channel combining. Also we will exam the impact of different interference model on the fairness and maximal throughput in wireless mesh networks with and without combining. Furthermore we will exam the improvement to fairness and maximal throughput in the wireless mesh network with channel-combining when increasing $C$ from 1 to $C_{\mathcal{M}}$. As we have discussed in the former section, the fairness and the maximal flow is solved by two linear programs. The result of the first linear program contributes to the input of the second one. In our simulations, let $\lambda_{1}$ be the fairness found from the first linear program LP-Flow-fairness. Then $\lambda_{1}$ is used as the input value of $\lambda_{0}$ in the second LP LP-Flow-throughput to solve the maximal throughput in the wireless mesh network. The wireless mesh network in this paper is generated randomly where the position of the node is chosen randomly. The network is generated under the constraint $n \pi r^{2} \geq c \log n$, where $n$ is the number of nodes, $r$ is the transmission radius and $c$ is some constant. The wireless mesh network is thus guaranteed to be connected with high probability, the reader can refer to [29] for proof of the connectivity constraint. We use $802.11 a$ for the link channel capacity in the wireless mesh network, which is same as [2]. The link channel capacity thus only depends on the distance between the two nodes at the end of the link. We set the link channel capacity as $54 \mathrm{Mbps}$ when the distance of the two end nodes is within 30 meters, the link channel capacity as $48 M b p s$ when the distance is within 32 meters, the link channel capacity as $36 \mathrm{Mbps}$ when the distance is within 37 meters, the link channel capacity as $24 M b p s$ when the distance is within 45 meters, the link channel capacity as $18 \mathrm{Mbps}$ when the distance is within 60 meters, the link channel capacity as $12 \mathrm{Mbps}$ when the distance is within 69 meters, the link channel capacity as $9 M b p s$ when the distance is within 77 meters, and the link channel capacity as $6 \mathrm{Mbps}$ when the distance is within 90 meters. Otherwise, if the distance of the two end nodes of the link is beyond 90 meters, we will set the link channel capacity as 0 , which makes sense because two nodes within their transmission radius may even not form a link due to limited power, environment disturb and so on. The traffic demand of each mesh node is randomly generated with a minimum of $4 M b p s$ and a maximum of $12 \mathrm{Mbps}$. Initially, the wireless mesh network is generated with 60 nodes and 8 gateways. The nodes are randomly dispersed in a square area of $500 \times 500$ square meters. The actual transmission radius of a node is randomly chosen between minimum value $r$ and the maximal value $R$. We vary the 
maximal number of NICs from 2 to 7 for a node while using 1 as them minimal number of the radio. The maximal number of channels varies also from 2 to 7 while the minimal number of channels is 2 , where the maximum (minimum) means that it is the maximal (minimal) value the radio or the channel can randomly choose.

In following subsections, due to limited space, we only present a subset of our results. When we vary channel numbers, our results showed the same trend, thus we select 2 of the results and comment on them and the same applies when varying number of radios.

\section{A. Impact of Multi-Channel}

In this simulation, we studied the affect of using multiple channels per radio on the fairness and maximal throughput in the wireless mesh network with channel-combining and without channel-combining configurations. In this simulation we fixed the maximum number of NICs to $1,2,3, \ldots, 7$ in separate simulation runs. In each simulation, we varied the maximum number of channels per radio from 2 to 7 and randomly assigned each radio number of channels between 2 and the maximum value at each simulation run. Figure 2(a) shows our results when maximum number of radios is 4 , each point in the figure is the average of 5 simulations. The figure shows that when channel-combining is performed it provides higher throughput and higher fairness compared with the case of no channel-combining, which is inline with our expectation. Also, the figure shows that increasing number of channels per radio increases the throughput and fairness. When we changed number of radios between 1 and 7 , our results showed the same trend; combining increased throughput and fairness compared to no combining and increasing number of channels per radio increases the fairness and throughput. Figure 2(a) shows that the fairness in channel-combining is almost twice of that without channel-combining while the throughput is twice more of that without channel-combining.

With number of radios increasing, the throughput and fairness does not increase as can be depicted from comparing the results in Figure 2(a) for 4 radios and 2(b) for 7 radios. This is because the network with higher radios (channels) does not guarantee to have more radios (channels) than that with smaller radios (channel), which is with high probability. After all, the network with higher maximal radio(channel) has a greater upper bound for the node to randomly choose the radios or channels.

\section{B. Impact of Multi-Radio}

In this simulation, we studied the affect of using multiple radios per node on the fairness and maximal throughput in the wireless mesh network with channel-combining and without channel-combining configurations. In this simulation we fixed the maximum number of channels to $2,3, \ldots, 7$ in separate simulation runs. In each simulation, we varied the maximum number of radios/node from 1 to 7 and randomly assigned each node number of radios between 1 and the maximum value at each simulation run. Figures 3 (a) \& (b) show our results when maximum number of channels is set to 4 and

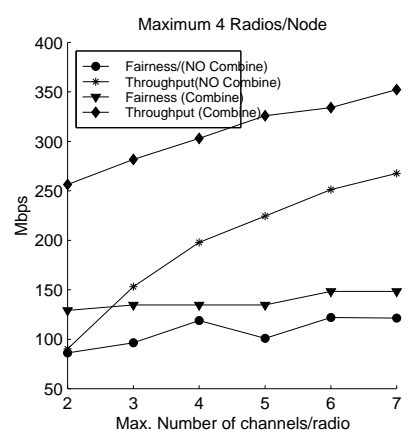

(a) 4 Radios

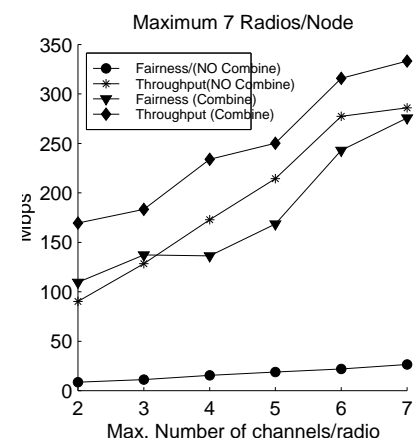

(b) 7 Radios
Fig. 2. Impact of multi-channel on fairness and throughput.

7 , each point in the figure is the average of 5 simulations. The figures show that when channel-combining is performed it provides higher throughput and higher fairness compared with the case of no channel-combining, which is consistent with our results discussed in section V-A for varying number of channels per radio. The figures, also, show that the throughput and the fairness measures increase until a certain point where they start bouncing up and down each time maximum number of radios/node increase. The reason behind the bouncing is that the actual number of radios assigned to each node is randomly generated for each simulation run, for the cases were the throughput and fairness decreases by increasing the maximum number of radios per node we compared the actual total number of radios in the network for all simulation runs. We found that when the maximum number of radios is 5 the actual total number of assigned radios for all nodes is less than that of the case when the maximum number of radios is 4. Therefore the number of available radios in the network is less hence (with fixed number of channels/radio) the probability of assigning non-conflicting channels for the interfering links decreased and that is why the fairness and throughput decreased. Same analysis applies when the maximum number of radios increased from 6 to 7 .

By comparing figures 3(a) and 3(b) we observe that the throughput decreases slower with more number of channels, i.e. the decrease is sharper with 4 channels than with 7 . This is due to the fact that more channels are assigned per radio which increases the probability of assigning non-conflicting channels to interfering links which increase throughput and fairness compared with the case of less number of channels/radio.

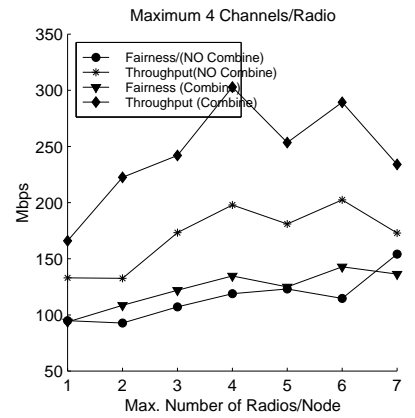

(a) 4 Channels

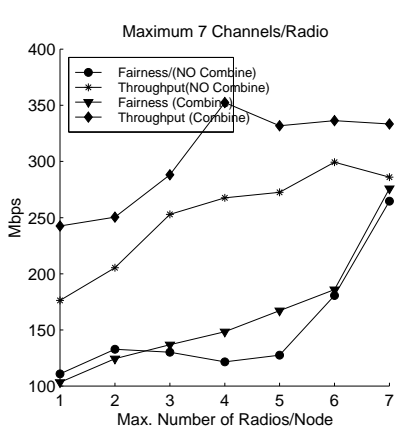

(b) 7 Channels
Fig. 3. Impact of multi-radio on fairness and throughput. 


\section{Impact of Interference Model}

In this simulation, we use the different interference models to study how the interference model can affect the fairness and maximum throughput in the wireless mesh network. We used a maximum number of 2 radios per node, and we measured the fairness and throughput of the mesh network, with channel-combining and without channel-combining configurations, where the maximum number of channels varies from 2 to 7 under the following interference models: RTS/CTS, fPrIM, TxIM, and PrIM. For interference models PrIM and TxIM we chose three different values for $\eta=\{0.0,0.1,0.3\}$ and conducted 5 simulations for each value.

The results of our simulations are shown in figures 4 and 5 . The figures show that different interference models result in different fairness and throughput. In the case without channelcombining, the network receives highest fairness and throughput under PrIM while receives lowest fairness and throughput under RTS/CTS model. In the case with channel-combining, the network receives highest fairness and throughput under PrIM model while receives lowest fairness under RTS/CTS model and lowest throughput under TxIM model.

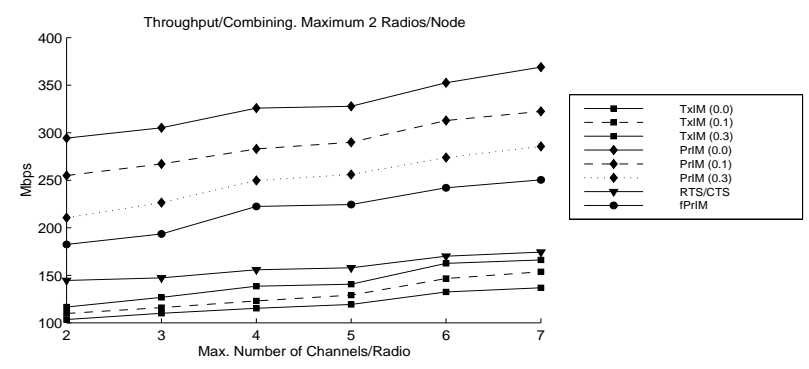

(a) Impact on Throughput

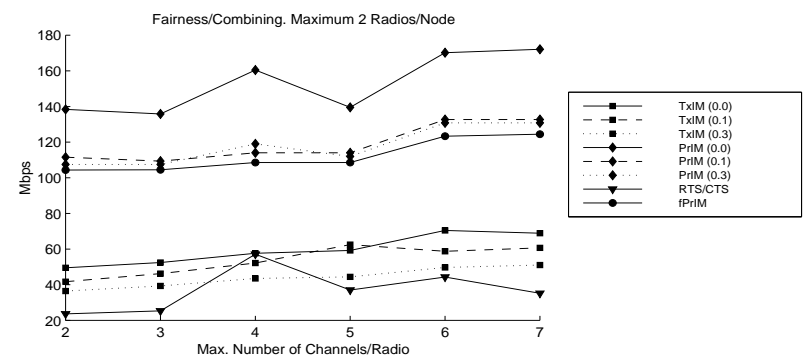

(b) Impact on Fairness

Fig. 4. Impact of interference models with channel-combining.

The different interference protocol models result in different fairness and throughput because the conflict graph is not the same for all models. Therefore, certain links may be scheduled at some time slot in one interference protocol model while they may not be scheduled under the other one. The less interference links one link has, the more probable it is to be scheduled and then the more fairness and throughput will be.

\section{Impact of $C$}

We then studied the improvement to fairness and maximal throughput in the wireless mesh network with channelcombining when increasing $C$ from 1 to $C_{\mathcal{M}}$. In this simulation we varied the maximum number of channels from 2

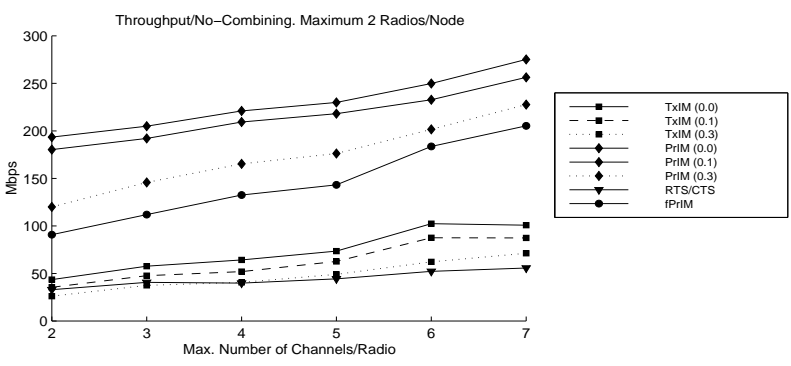

(a) Impact on Throughput

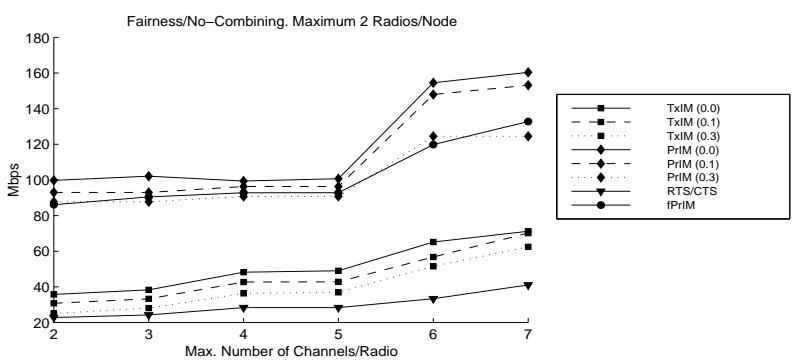

(b) Impact on Fairness

Fig. 5. Impact of interference models without channel-combining.

to 4 and the maximum number of radios from 1 to 2 . For the different combinations of (number of radios, number of channels) we changed $C$ from 1 to 10 in 1 step increment. Figures 6 (a) \& (b) show our results when maximum number of channels is 4 and maximum number of radios is 1 or 2 respectively, each point in the figure is the average of 5 simulations. We observed that fairness and throughput increase with increasing $C$, as we expected, and at a certain $C_{0}$ the fairness and throughput will reach the maximum demand of all nodes and will not increase with increasing $C$, in figures 6 (a) and (b) $C_{0}=6$ for 1 radio configuration and $C_{0}=5$ for 2 radios configuration. We also found that $C_{0}$ decreases by increasing number of radios per node, but increasing number of channels does not change the value of $C_{0}$.

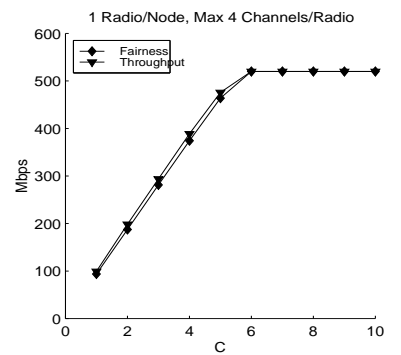

1 Radio, 4 Channels

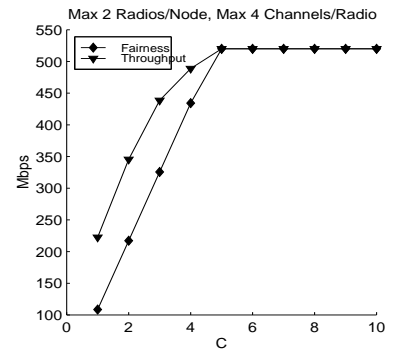

(b) 2 Radio, 4 Channels
Fig. 6. Impact of changing $C$ on fairness and throughput.

\section{RELATED WORK}

A number of results [3]-[5], [14], [22]-[26] have been proposed recently for channel assignment and/or routing in multichannel and multi-radio wireless networks. When multiple channels are available, one class of work is to enable only one network interface card to operate in multiple channels. In majority schemes along this line, e.g., [3], [4], [20], using a single interface, frequent channel switching introduces extra 
overhead, and nodes are inherently limited to one reception or transmission at a given time. Another line of work is to use multiple interfaces available at each wireless nodes. Among these methods, [1], [2], [5], [23] use a static channel assignment. Raniwala et al. [23] considered the joint problem of channel assignment and routing in static mesh networks. They assume that long-term traffic load between source and destination pairs is known a priori. More recently, they proposed a distributed algorithm [22]. Draves et al. [5] proposed a new routing metric when nodes have multiple interface cards. They assume that each edge is assigned a fixed channel, but they do not consider the channel assignment problem.

Another key problem for improving the throughput of a multi-radio multi-channel multi-hop network is the scheduling of node activities. Scheduling has been studied extensively [18], [19], [21] based on coloring. Wang et al. [27] recently proposed efficient centralized and distributed link scheduling algorithms to minimize time-span under PrIM and fPrIM for single-channel single-radio wireless networks.

Optimizing throughput, using a joint approach of multi-path routing, link scheduling, and channel assignment, has been studied for various network models, e.g., [2], [10]-[13]. Kodialam and Nandagopal [11] studied the effect of interference on the achievable rate region in multi-hop wireless networks. They treated the interference models as linear constraints and solved the flow problem using linear program. In [10], the same authors considered the problem of jointly routing the flows and scheduling transmissions to achieve a given rate vector using the protocol interference model. They developed necessary and sufficient conditions for the achievable rate vector. The scheduling problem is solved as a graph edgecoloring problem using existing greedy algorithms. In [12], they extended their work to the multi-radio multi-channel wireless mesh networks. They used a linear programming solution to assign channels to links and also schedule the time slots in which each link and channel is active, using greedy approach. The main differences between [10]-[12] and our results here are that they only consider primary interference, UDG network model with single channel. Kumar et al. [13] considered the throughput capacity of wireless networks for unicast for PrIM and TxIM interference models. They developed analytical performance evaluation models and distributed algorithms for routing and scheduling which incorporate fairness, energy and dilation (path-length) requirements to maximize network throughput. They also only considered the single channel model. Alicherry et al. [2] established necessary and sufficient conditions under which interference free link communication schedule can be obtained and designed a simple greedy algorithm to compute such a schedule. They showed that their algorithm for the joint channel assignment, routing and scheduling problem is a constant factor approximation algorithm. Notice that the studied network in [2] is restricted to be a UDG, i.e., the uniform interference range is assumed to be a fixed multiple of the uniform communication range. They also assume that the network will adopt a fixed channel assignment. Paper [27] only consider link scheduling in single channel networks without addressing flow assignment and multi-channels. We could not find any results that considered the channel combining to improve the throughput.

\section{CONCLUSION}

We studied the problem of cross-layer multi-path routing, interference-aware link scheduling, dynamic channel assignment for a multi-channel, multi-radio, multi-hop wireless network with and without channel-combining to maximize the network fairness or throughput (given traffic demands). We presented efficient algorithms that achieve fairness or throughput within a constant factor of the optimum. Our theoretical performance guarantees rely on very weak requirements of the wireless network. We also conducted extensive simulations to study the performances of our algorithms and study the impact of channel-combining to maximize the fairness or throughput of the network. Our results showed that 1) channelcombining indeed increases fairness and throughput, 2) when channel-combining is supported, increasing number of radios will increase the fairness and throughput until some threshold and then decrease the fairness and throughput. A number of challenging questions are left for future research, e.g., study the dynamic link channel scheduling by taking into account the channel-combining cost in a dynamic environment where the traffic load and capacity on links fluctuates.

\section{REFERENCES}

[1] Adya, A., Bahl, P., Padhye, J., Wolman, A., And Zhou, L. A multi-radio unification protocol for ieee 802.11 wireless networks. Microsoft Technical Report, MSR-TR-2003-41, June 2003.

[2] Alicherry, M., Bhatia, R., AND Li, L. E. Joint channel assignment and routing for throughput optimization in multi-radio wireless mesh networks. In ACM MobiCom (2005), pp. 58-72.

[3] BAhl, V., Chandra, R., And Dunagan, J. SSCH: Slotted seeded channel hopping for capacity improvement in ieee 802.11 ad-hoc wireless networks. In Proc. ACM Mobicom 2004.

[4] Chandra, R., Bahl, P., And Bahl, P. Multinet: Connecting to multiple ieee 802.11 networks using a single wireless card. In IEEE INFOCOM 2004.

[5] Draves, R., PADhye, J., AND Zill, B. Routing in multi-radio, multihop wireless mesh networks. In ACM Mobicom 2004.

[6] Grossglauser, M., And Tse, D. N. C. Mobility increases the capacity of ad-hoc wireless networks. In INFOCOM (2001), pp. 13601369.

[7] Gupta, P., And Kumar, P. Capacity of wireless networks. IEEE Transactions on Information Theory, vol. IT-46, no.2, pp.388-404, 2000.

[8] Jain, K., Padhye, J., Padmanabhan, V. N., And QiU, L. Impact of interference on multi-hop wireless network performance. In $A C M$ MobiCom (2003), pp. 66-80.

[9] Kilpatrick, J. A., CYr, R. J., ORg, E. L., AND DAwE, G. New SDR architecture enables ubiquitous data connectivity. RF Design Magazine, http://rfdesign.com/mag/601RFDF3.pdf (January 2006), 34-38.

[10] Kodialam, M., AND NAndagopal, T. Characterizing achievable rates in multi-hop wireless networks: the joint routing and scheduling problem. In ACM MobiCom (2003), pp. 42-54.

[11] Kodialam, M., and Nandagopal, T. The effect of interference on the capacity of multi-hop wireless networks. In Proceedings of IEEE Symposium on Information Theory (2004).

[12] Kodialam, M., And Nandagopal, T. Characterizing the capacity region in multi-radio multi-channel wireless mesh networks. In $A C M$ MobiCom (2005), pp. 73-87.

[13] Kumar, V. S. A., Marathe, M. V., Parthasarathy, S., And SRINIVASAN, A. Algorithmic aspects of capacity in wireless networks. SIGMETRICS Perform. Eval. Rev. 33, 1 (2005), 133-144.

[14] Kyasanur, P., AND VAIDYA, N. H. Routing in multi-channel multiinterface ad hoc wireless networks. CS dept. UIUC Technical Report, December 2004.

[15] KYASANUR, P., AND VAIDYA, N. H. Capacity of multi-channel wireless networks: impact of number of channels and interfaces. In $A C M$ MobiCom (2005), pp. 43-57. 
[16] Kyasanur, P., And Vaidya, N. H. Capacity of MultiChannel Wireless Networks: Impact of Channels, Interfaces and Interface Switching Delay, Technical Report, October 2006

[17] Li, J., Blake, C., Couto, D. S. J. D., Lee, H. I., AND Morris, R. Capacity of ad hoc wireless networks. In ACM Mobile Computing and Networking (2001), pp. 61-69.

[18] LiU, R., AND Lloyd, E. L. A distributed protocol for adaptive link scheduling in ad-hoc networks. In Proceedings IASTED Int. Conf. on Wireless and Optical Comm. (WOC2001) (2001).

[19] Moscibroda, T., ANd Wattenhofer, R. Coloring unstructured radio networks. In ACM SPAA (2005), pp. 39-48.

[20] NASIPURI, A., ZhUANG, J., AND DAS, S. R. A multichannel csma mac protocol for multihop wireless networks. In IEEE WCNC, (1999).

[21] Ramanathan, S. A unified framework and algorithm for channel assignment in wireless networks. Wireless Network 5, 2 (1999), 81-94.

[22] Raniwala, A., AND Chiueh, T. Architecture and algorithms for an ieee 802.11-based multi-channel wireless mesh network. In INFOCOM 2005.

[23] Raniwala, A., Gopalan, K., And Chiueh, T. Centralized channel assignment and routing algorithms for multi-channel wireless mesh networks. ACM MCCR 8, 2 (2004).

[24] So, J., AND VAIDYA, N. Multi-channel mac for ad hoc networks: Handling multi-channel hidden terminals using a single transceiver. In ACM MobiHoc 2004.

[25] So, J., AND VAIDYA, N. H. Routing and channel assignment in multichannel multi-hop wireless networks with single-nic devices. CS dept. UIUC Technical Report, December 2004.

[26] WANG, J., FANG, Y., AND WU, D. A power-saving multi-radio multichannel mac protocol for wireless local area networks. In IEEE INFOCOM (2006).

[27] Wang, W., Wang, Y., Li, X.-Y., Song, W.-Z., ANd Frieder, O. Efficient interference aware tdma link scheduling for static wireless mesh networks. In ACM MobiCom (2006).

[28] Yi, S., Pei, Y., And Kalyanaraman, S. On the capacity improvement of ad hoc wireless networks using directional antennas. In $A C M$ MobiHoc (2003), pp. 108-116.

[29] Gupta, P., AND Kumar, P.R. Critical power for asymptotic connectivity. In the 37th IEEE Conference on Decision and Control (1998).

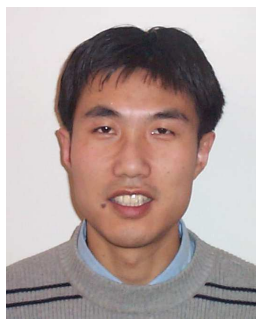

Dr. Xiang-Yang Li has been an Associate Professor since 2006 and Assistant Professor of Computer Science at the Illinois Institute of Technology from 2000 to 2006. He was a visiting professor of Microsoft Research Asia from May 2007 to August 2008. He hold MS (2000) and PhD (2001) degree at Computer Science from University of Illinois at Urbana-Champaign. He received B.Eng. at Computer Science and Bachelor degree at Business Management from Tsinghua University, P.R. China in 1995. His research interests span wireless ad hoc and sensor networks, non-cooperative computing, computational geometry, and algorithms. He was a guest editor of special issues for ACM Mobile Networks and Applications, IEEE Journal on Selected Areas in Communications.

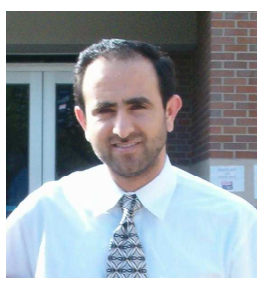

Ashraf Nusairat is Computer Science PhD student at Illinois Institute of Technology and works for Motorola Inc as Wireless Broadband System Architect. He received his MS in Computer Science in 1999 from The University of Akron - $\mathrm{OH}$ and received his Bachelor degree in Computer Engineering in 1993 from Jordan University of Science and Technology - Jordan. His research interests are in the areas of wireless broadband networks, wireless mesh networks and wireless ad-hoc networks.

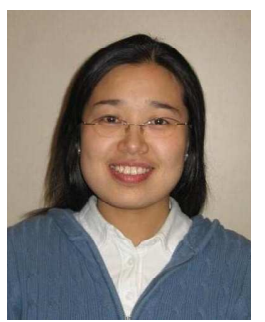

Yanwei $\mathbf{W u}$ is a Computer Science $\mathrm{PhD}$ student at Illinois Institute of Technology. She received B.Eng and M.E. from TianJing University, P.R.China, 1998 and 2003 respectively. Her research interests span wireless networks, game theoretical study of networks, optimization in mesh network, energy efficiency, security in wireless network. She also researched on agent based modeling as a Research Aide in Argonne National lab in 2007.

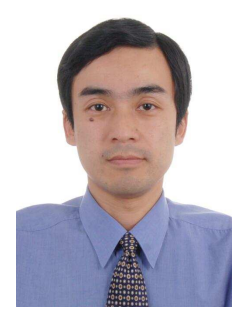

Dr. JiZhong zhao is a Professor of Computer Science and Technology Department, Xi'an Jiaotong University, China. He received his BS degree and MS degree in Mathematic Department from Xi'an Jiaotong University, China. He received a Ph.D. degree in Computer Science, focus on Distributed System, from Xi'an Jiaotong University in 2001. His research interests include computer software, pervasive computing, distributed systems, network security. He is a member of IEEE computer society, and a member of ACM.

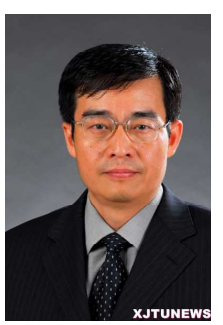

Dr. Yong Qi has been a Professor since 2001 and Associate Professor of Computer Science and Technology Department at Xian Jiaotong University from 1996 to 2001P.R. China. He hold MS (1996) and $\mathrm{PhD}$ (2001) degree at Computer Science from Xian Jiaotong University.His research interests span sensor networks, operating system, distributed middleware, and services computing. He is a member of IEEE.

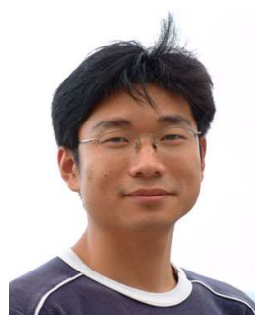

Dr. Xiaowen Chu received his B.Eng. degree in the Computer Science from Tsinghua University, P. R. China, in 1999, and the Ph.D. degree in the Computer Science from the Hong Kong University of Science and Technology in 2003. He is currently an Assistant professor in the Department of Computer Science, Hong Kong Baptist University. He is the program co-chair of the 3rd IEEE International Symposium on Security in Networks and Distributed Systems, and vice-Chair of the 10th IEEE International Conference on High Performance Computing and Communications. His research interests include Wireless Networks, Optical Networks, Internet Security, P2P, Multimedia, and Web Server Performance. He is on the Editor Board of MDPI Journal of Algorithms.

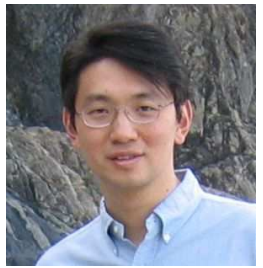

Dr. Yunhao Liu received his BS degree in Automation Department from Tsinghua University, China, in 1995, and an MA degree in Beijing Foreign Studies University, China, in 1997, and an MS and a $\mathrm{Ph} . \mathrm{D}$. degree in Computer Science and Engineering at Michigan State University in 2003 and 2004, respectively. He is now an assistant professor in the Department of Computer Science and Engineering at Hong Kong University of Science and Technology. His research interests include sensor networking, pervasive computing, and peer-to-peer computing. $\mathrm{He}$ is a senior member of the IEEE Computer Society. 